\title{
Contribution to the knowledge of Ichneumoninae of Siberia and Far East Russia (Hymenoptera, Ichneumonidae)
}

\author{
With 44 figures
}

Matthias Riedel ${ }^{1}$

\author{
${ }^{1}$ Zoologische Staatssammlung München, Münchhausenstraße 21, 81247 München, Germany. - mamaflo.riedel@t-online.de \\ Published on 2020-12-01 \\ DOI:10.21248/contrib.entomol.70.2.365-386 \\ LSID urn:lsid:zoobank.org:pub:0E34351E-B1AE-4334-AB1C-29C38CEF6318
}

\begin{abstract}
In this survey, distributional records of 81 Ichneumoninae from Siberia and Far East Russia are given. 44 of them are newly reported from Siberia and/or Far East Russia and 25 species have not been found in the East Palaearctic region before.

Eleven species are described as new: Aoplus ocellator spec. nov., Ectopius sibiricus spec. nov., Hoplismenus flagellator spec. nov., Ichneumon brevimaculatus spec. nov., Ichneumon flavocoxalis spec. nov., Ichneumon flavotibialis spec. nov., Ichneumon megextenorius spec. nov., Ichneumon pilocephalus spec. nov., Ichneumon taegeri spec. nov., Monontos baikalensis spec. nov. and Protichneumon nigriventris spec. nov. One taxon is described as a new subspecies: Chasmias paludator sibiricus subspec. nov.

For Coelichneumon septenus Townes et al., 1965 a new female is described. The genus Monontos UcHIDA is transferred from the tribe Heresiarchini to the Ichneumonini.
\end{abstract}

\section{Nomenclatural acts}

Aoplus ocellator spec. nov. - urn:lsid:zoobank.org:act:48A8BD7B-482E-46DB-A330-DD5EEB39DE4C

Ectopius sibiricus spec. nov. - urn:lsid:zoobank.org:act:AD4C044E-A817-41C5-B24B-83085E159819

Hoplismenus flagellator spec. nov. - urn:lsid:zoobank.org:act:675CC203-04A9-4B23-9247-880562A8B0C4

Ichneumon brevimaculatus spec. nov. - urn:lsid:zoobank.org:act:32A6E211-9DC7-4E78-A7C4-D7C1FD8F1A6C

Ichneumon flavocoxalis spec. nov. - urn:lsid:zoobank.org:act: F0B7571F-F53D-4F5E-AEF7-E8E0DEC72F1D

Ichneumon flavotibialis spec. nov. - urn:lsid:zoobank.org:act:07E8B9DD-3819-4F13-B988-5FAC6CBA25C3

Ichneumon megextenorius spec. nov. - urn:lsid:zoobank.org:act:3F921DA4-7285-4328-85AB-C32096485FA8

Ichneumon pilocephalus spec. nov. - urn:lsid:zoobank.org:act:FCB03748-C2BF-47B5-9439-397B6E944973

Ichneumon taegeri spec. nov. - urn:lsid:zoobank.org:act:2EFB6CDD-10E9-4D81-8639-D8E54F68DC08

Monontos baikalensis spec. nov. - urn:lsid:zoobank.org:act:FD6CE0F9-611F-4590-9511-6DBABF35EE9A

Protichneumon nigriventris spec. nov. - urn:lsid:zoobank.org:act:98F723A9-B2A8-44D8-B118-83F88FAEAE7E

\section{Key words}

Hymenoptera, Ichneumonidae, Ichneumoninae, new species, Siberia, Far East Russia 


\section{Zusammenfassung}

In dieser Arbeit werden Fundorte von 81 Ichneumoninae aus Sibirien oder Fernost-Russland aufgeführt. 44 von ihnen sind bisher nicht aus diesen Regionen nachgewiesen worden, 25 Arten sind Neunachweise für die gesamte ostpaläarktische Fauna.

Elf Arten werden neu beschrieben: Aoplus ocellator spec. nov., Ectopius sibiricus spec. nov., Hoplismenus flagellator spec. nov., Ichneumon brevimaculatus spec. nov., Ichneumon flavocoxalis spec. nov., Ichneumon flavotibialis spec. nov., Ichneumon megextenorius spec. nov., Ichneumon pilocephalus spec. nov., Ichneumon taegeri spec. nov., Monontos baikalensis spec. nov. und Protichneumon nigriventris spec. nov. Außerdem wird eine neue Unterart beschrieben: Chasmias paludator sibiricus subspec. nov.

Für Coelichneumon septenus Townes et al., 1965 wird ein neues Weibchen beschrieben. Die Gattung Monontos UchIDA wird von den Heresiarchini zu den Ichneumonini transferriert.

\section{Introduction}

The subfamily Ichneumoninae is a very large group of Ichneumonidae (Hymenoptera) which are known as endoparasitoids of Lepidoptera (BROAD et al., 2018). Although this subfamily includes many large and colorful species, our knowledge on taxonomy, distribution and biology of many Ichneumoninae is still incomplete.

In a recent catalogue (LELEJ, 2012), 251 species of Ichneumoninae were listed for Russian Far East, and 760 species are known from the entire East Palaearctic region so far (Yu et al., 2016), but we can expect many more species of this subfamily of Ichneumonidae in the East Palaearctic region.

In the last years, I was able to study several collections of Siberian Ichneumoninae. In a first recent publication (RIEDEL, 2018), 20 species of the large genus Ichneumon LinNAEUs were described as new. Here, I present a second survey containing several genera of the Ichneumoninae from Siberia and Far East Russia.

\section{Material and Methods}

For this study, I had the opportunity to determine East Palaearctic Ichneumoninae from the Senckenberg Deutsches Entomologische Institut, Müncheberg/ Germany (SDEI), the Zoologische Staatssammlung München/Germany (ZSM), the Naturkundemuseum Erfurt/Germany (Erfurt), the National Museums of Scotland, Edinburgh/Scotland (EDI), the Natural History Museum, London/United Kingdom (NHM) and my own collection (coll. RIEDEL).

For the descriptions below, morphological terms follow BROAD et al. (2018). The distributional records were taken from the catalogues of LELEJ (2012) and YU et al. (2016).

For the measurements the following relations were used: Length of $1^{\text {st }}$ flagellomere was measured in lateral view (length without anellus); width of gena and eye were measured from lateral view, and length and width of hind femur in lateral view. For the punctuation of body parts the following definitions were used: very scattered
- distance of punctures $>2 \mathrm{x}$ their diameter; scattered distance $1-2 \mathrm{x}$ their diameter; rather dense - distance about as their diameter; dense - diameter of punctures larger than their distance.

For the measurements an Olympus SZX 7 stereo microscope with dividing eyepiece was used. The figures were taken with an Olympus SC 30 CCD-camera using the AnalySIS getIT and Helicon Focus Pro softwares and processed with the Microsoft Office Picture manager.

\section{List of species}

Tribe Phaeogenini

Aethecerus nitidus (WeSMAEL, 1845)

Material: East Siberia, Irkutsk region, Lisikha, 1 o 2007 07-21, leg. O. Berlov (coll. Riedel).

Distribution: Palaearctic region, known from Siberia (SiYTAN, 1977).

\section{Colpognathus celerator (GRAVENHORST, 1807)}

Material: East Siberia, Irkutsk region, $5 \mathrm{~km}$ Southeast of Ust-Orda, 1 \& 2007-06-29, leg. O. Berlov (coll. Riedel).

Distribution: Palaearctic region, known from Siberia (SiYtAn, 1977).

\section{Colpognathus divisus Thomson, 1891}

Material: East Siberia, Irkutsk region, Lisikha, 1 o 2007 07-20, leg. O. Berlov (coll. Riedel).

Distribution: Palaearctic region, known from Siberia (Diller \& SCHÖNITZER, 2003). 


\section{Diadromus subtilicornis (GRAVENHORST, 1829)}

Material: East Siberia, Irkutsk region, $5 \mathrm{~km}$ Southeast of Ust-Orda, 1 i 2007-06-29, leg. O. Berlov (coll. Riedel).

Distribution: Holarctic region, known from Siberia (SiYtan, 1977).

\section{Dirophanes fulvitarsis (WESMAEL, 1845)}

Material: East Siberia, 11 km East of Irkutsk, 1 ๆ $2007-$ 08-28, leg. O. Berlov (coll. Riedel).

Distribution: Palaearctic region, known from Siberia (SIYTAN, 1977).

\section{Dirophanes mysticus (WESMAEL, 1855)}

Material: East Siberia, $11 \mathrm{~km}$ East of Irkutsk, 1 o $^{\top} 2007-$ 08-28, $10 \mathrm{~km}$ East of Irkutsk, 1 o $^{\star} 18$.VIII.2007, leg. O. Berlov (coll. Riedel).

Distribution: Palaearctic region, known from Mongolia, new record for Siberia.

\section{Tycherus ophtalmicus ophtalmicus (WESMAEL, 1845)}

Material: East Siberia, Irkutsk region, Lisikha, 1 ơ 200508-04, leg. O. Berlov (coll. Riedel).

Distribution: Holarctic region, known from Japan and Sakhalin (UCHIDA, 1936).

\section{Heterischnus filicornis (GRAVENHORST, 1829)}

Material: East Siberia, lake Baikal, Marytui, 1 우 2007-08 22, leg. O. Berlov (coll. Riedel).

Distribution: West Palaearctic region, new record for the East Palaearctic region.

\section{Mevesia arguta (WESMAEL, 1845)}

Material: East Siberia, Irkutsk, 1 o $^{*}$ 2006-08-16, leg. O. Berlov; East Siberia, Irkutsk region, Lisikha, 1 우 200508-4, leg. O. Berlov (coll. Riedel).

Distribution: West Palaearctic region, new record for the East Palaearctic region.

\section{Phaeogenes melanogonos (GMELIN, 1790)}

Material: East Siberia, $10 \mathrm{~km}$ East of Irkutsk, $20^{\star} \sigma^{\star}$ 200707-06, leg. O. Berlov (coll. Riedel).

Distribution: Palaearctic region, known from Siberia (SiYTAN, 1977).

\section{Phaeogenes nigridens WeSMAEL, 1845}

Material: East Siberia, $10 \mathrm{~km}$ East of Irkutsk, $20^{\star} o^{\star}$ 200707-07, leg. O. Berlov (coll. Riedel).

Distribution: Holarctic region, known from Japan (Townes et al., 1965), new record for Siberia.

\section{Tycherus socialis (RATZEBURG, 1852)}

Material: East Siberia, lake Baikal, Backalo-Lenskiy reserve, 1 o 2005-07-10, leg. O. Berlov (coll. Riedel).

Distribution: West Palaearctic region, new record for the East Palaearctic region.

\section{Tribe Platylabini}

\section{Ectopius sibiricus spec. nov. urn:Isid:zoobank.org:act:AD4C044E-A817-41C5-B24B-83085E159819 (Figs 15-17)}

Holotype: o "Russia (Far East), Primorsky Kray, 3 m,

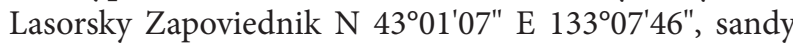
coast 13.6.-6.7.01 Malaise trap, M. Kuhlmann” (EDI).

Description: + . Body length $5 \mathrm{~mm}$. Flagellum with 30 flagellomeres, distinctly lanceolate; $1^{\text {st }}$ flagellomere $4 \mathrm{x}$ longer than wide; widest flagellomeres c. $2 \mathrm{x}$ wider than long. Temple moderately and roundly narrowed behind eye (Fig. 1). Frons with fine punctures, otherwise smooth and shining. Face closely punctate. Clypeus more sparsely punctate, intervals smooth and shining. Gena $0.8 \mathrm{x}$ as wide as the compound eye. Malar space $0.6 \mathrm{x}$ as long as width of mandibular base. Mandible with two teeth; upper tooth distinctly longer than lower one. Genal carina reaching hypostomal one close to mandibular base, both carinae low.

Notaulus not impressed. Mesoscutum with dense and coarse punctures, intervals smooth and shining. Mesopleuron and metapleuron densely punctate, smooth. Sternaulus indistinct, epicnemial carina narrow. Scutellum moderately convex, slightly wider than long, with lateral carina in its basal half. Area superomedia about rectangular, $2.5 \mathrm{x}$ wider than long. Costula distinct medially, obsolete laterally, reaching area superomedia in its middle. Area petiolaris rugose, \pm matte. Spiracles of 
propodeum long-oval. Outer corner of area dentipara without apophysis. Hind femur 4.1x longer than wide. Hind metatarsus c. $0.5 \mathrm{x}$ as long as hind tibia and about as long as combined length of $2^{\text {nd }}$ to $4^{\text {th }}$ hind tarsomeres. Claws not pectinate (in contrast to E. rubellus). Areolet pentagonal; $2^{\text {nd }}$ recurrent vein distal to its middle. Nervulus interstitial. Nervellus of hind wing interstitial, intercepted in its apical 3/4.

Metasoma amblypygous. Petiolus wider than high, rather long. Postpetiolus moderately widened, c. $0.7 \mathrm{x}$ as wide as the distance of outer hind corners of the areae dentiparae, with weak dorsal carina. Median field of postpetiolus almost smooth and shining, lateral field rugulose. $2^{\text {nd }}$ tergite $1.25 \mathrm{x}$ wider than long. Gastrocoelus triangularly impressed, smooth (Fig. 1). Thyridium indistinct. $2^{\text {nd }}$ tergite with dense coarse punctures, intervals smooth and shining. Punctures of $3^{\text {rd }}$ tergite somewhat shallower, with microsculpture laterally, but shining. $4^{\text {th }}$ tergite with very superficial punctures and with microsculpture. Ovipositor sheath narrow (as in E. rubellus), conically narrowed to apex.

Color: Black. Basal flagellomeres reddish-brown, flagellomeres 8-13 with ivory stripes. Spots on frontal orbit, apical 0.4 of malar space, mandible centrally, and spot on subtegular ridge ivory. Scutellum reddish centrally. Metasoma black; $2^{\text {nd }}$ to $7^{\text {th }}$ tergites with very narrow yellowish hind margins. Coxae and trochanters black; fore and mid trochanters with yellow spots frontally. Legs otherwise red; hind femur red, darkened in apical 0.15; hind tibia yellowish-red in basal 0.6 and black in apical 0.4 ; hind tarsomeres brownish, basally yellowish. Pterostigma ochreous.

ơ: unknown.

Remark: The new species resembles Ectopius rubellus (GMELIN) in general appearence and coloration, but differs by the form of the antenna, temple and $2^{\text {nd }}$ tergite. The claws have small teeth in Ectopius rubellus (GMELIN), but are not pectinate in E. sibiricus spec. nov.

\section{Pristicerops laetepictus (CosTA, 1864)}

Material: East Siberia, Irkutsk region, $5 \mathrm{~km}$ East of Pivovarikha, 1 क 2007-07-16, leg. O. Berlov (coll. Riedel).

Distribution: West Palaearctic region, new record for the East Palaearctic region.

\section{Platylabus iridipennis (GRAVENHORST, 1829)}

Material: East Siberia, Irkutsk region, Lisikha, 1 우 200408-17, leg. O. Berlov (coll. Riedel).

Distribution: Palaearctic region, known from Japan (UchidA, 1936), new record for Siberia.

\section{Platylabus opaculus Thomson, 1888}

Material: East Siberia, $10 \mathrm{~km}$ East of Irkutsk, 1 ? 200508-22, leg. O. Berlov (coll. Riedel).

Distribution: Holarctic region, new record for Siberia.

\section{Platylabus orbitalis (GRAVENHORST, 1829)}

Material: East Siberia, 10 km East of Irkutsk, 1 ㅇ 200408-09, leg. O. Berlov (coll. Riedel).

Distribution: West Palaearctic region, new record for the East Palaearctic region.

\section{Platylabus pallidens WeSMAEL, 1853}

Material: Russia Far East, Primorskiy Kray, Lazovski Zapovednik, c. $170 \mathrm{~km}$ East of Vladivostok, Ta-Chingousa, $43^{\circ} 01^{\prime} 07^{\prime \prime} \mathrm{N} 134^{\circ} 07^{\prime} 46^{\prime \prime} \mathrm{E}, 0 \mathrm{~m}, 1$ o $2001-08-31$ to 2001-09-16, sandy coast, Malaise trap 495, leg. M. Quest (NHM).

Distribution: West Palaearctic region, new record for the East Palaearctic region.

\section{Platylabus rufus WeSMAEL, 1845}

Material: Russia Far East, Primorskiy Kray, Lazovski Zapovednik, c. $170 \mathrm{~km}$ East of Vladivostok, Lazo, $43^{\circ} 30^{\prime} 30^{\prime \prime} \mathrm{N} 134^{\circ} 06^{\prime} 58^{\prime \prime} \mathrm{E}, 1353 \mathrm{~m}, 1$ o $^{\top} 2001-09-07$ to 2001-09-28, subalpine, Malaise trap 496, leg. M. Quest (NHM).

Remark: A dark specimen with black mesosoma, reddish face, brown metasoma, and reddish apical margins of tergites; otherwise typical (RIEDEL, 2008a: 151-152).

Distribution: Palaearctic region, known from Japan (Townes et al., 1965), new record for Far East Russia.

\section{Tribe Eurylabini}

\section{Eurylabus quadratus UCHIDA, 1926}

Material: Russia Far East, Primorskiy Kray, Lazovski Zapovednik, c. $170 \mathrm{~km}$ East of Vladivostok, Ta-Chingousa, $43^{\circ} 01^{\prime} 07^{\prime \prime} \mathrm{N} 134^{\circ} 07^{\prime} 46^{\prime \prime} \mathrm{E}, 0 \mathrm{~m}, 1$ o $^{\top} 2001-08-31$ to 2001-09-16, sandy coast, Malaise trap 495, leg. M. Quest (NHM).

Distribution: Japan and Korea, new record for Russia Far East. 
Tribe Listrodromini

Neotypus nobilitator (GraveNHORST, 1807)

Material: Russia Far East, Primorskiy Kray, Lazovski Zapovednik, c. $170 \mathrm{~km}$ East of Vladivostok, Ta-Chingousa, $43^{\circ} 01^{\prime} 07^{\prime \prime} \mathrm{N} 134^{\circ} 07^{\prime} 46^{\prime \prime} \mathrm{E}, 0 \mathrm{~m}, 1$ 우 2001-08-31 to 2001-09-16, sandy coast, Malaise trap 495, leg. M. Quest (NHM).

Distribution: Holarctic and Oriental regions, new record for Russia Far East.

\section{Tribe Heresiarchini}

\section{Coelichneumon assimilis (KOKUJEV, 1904)}

Material: Russia Far East, Primorskiy Kray, Lazovski Zapovednik, c. $170 \mathrm{~km}$ East of Vladivostok, Korpad, $43^{\circ} 17^{\prime} 15^{\prime \prime} \mathrm{N} 134^{\circ} 07^{\prime} 10^{\prime \prime} \mathrm{E}, 526 \mathrm{~m}, 3$ o $^{\text {त }} \mathrm{o}^{\mathrm{T}} 2001-06-16$ to 2001-06-25, Malaise trap 466, leg. M. Quest (NHM); Russia Far East, Primorskiy Kray, Lazovski Zapoved-

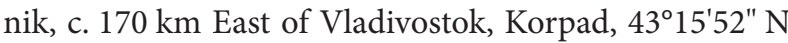
$134^{\circ} 07^{\prime} 45^{\prime \prime}$ E, 174 m, 1 ơ 2001-06-16 to 2001-06-25, flood plain, Malaise trap 467, leg. M. Quest (NHM).

Distribution: East Palaearctic region, known from Siberia (RIEDEL, 2008b).

\section{Coelichneumon bivittatus (Matsumura, 1912}

Material: Russia Far East, Primorskiy Kray, Lazovski Zapo-

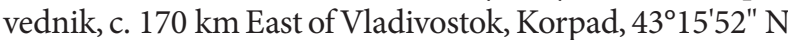
$134^{\circ} 07^{\prime} 45^{\prime \prime}$ E, 174 m, 1 ơ 2001-06-20 to 2001-06-27, flood plain, Malaise trap 469, leg. M. Quest (NHM).

Distribution: China, Japan and Korea (Yu et al., 2016), new record for Russia Far East.

\section{Coelichneumon cyaniventris (WESMAEL, 1845)}

Material: Russia Far East, Primorskiy kray, Ryazanovka, $14 \mathrm{~km}$ Southwest of Slavyanka, $42^{\circ} 48^{\prime} \mathrm{N} 131^{\circ} 12^{\prime} \mathrm{E}, 50 \mathrm{~m}$, 1 ㅇ 1993-06-16, leg. A. Taeger (SDEI).

Distribution: Palaearctic region, known from Russia Far East (RIEDEL, 2012).

\section{Coelichneumon eburnifrons (WESMAEL, 1857)}

Material: Russia Far East, Primorskiy Kray, Lazovski Zapo-

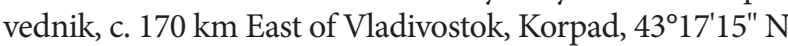
$134^{\circ} 07^{\prime} 10^{\prime \prime}$ E, $526 \mathrm{~m}, 1$ o $^{\mathrm{T}} 2001-05-13$ to 2001-06-5, mountain top, Malaise trap 447, leg. M. Quest (NHM).
Distribution: Holarctic region, known from Korea, new record for Russia Far East.

\section{Coelichneumon falsificus (WeSMAEL, 1845)}

Material: Russia Far East, Primorskiy Kray, Lazovski Zapovednik, c. $170 \mathrm{~km}$ East of Vladivostok, Korpad, $43^{\circ} 15^{\prime} 52^{\prime \prime} \mathrm{N} 134^{\circ} 07^{\prime} 45^{\prime \prime} \mathrm{E}, 174 \mathrm{~m}, 10^{\top} 2001-06-20$ to 2001-06-27, flood plain, Malaise trap 469, leg. M. Quest (NHM).

Remark: Nervulus strongly postfurcal, $2^{\text {nd }}$ recurrent vein proximal to middle of areolet, pterostigma black, otherwise typical (see RIEDEL, 2012: 1522-1523).

Distribution: Palaearctic region, known from Siberia (Meyer, 1933).

\section{Coelichneumon gargawensis UCHIDA, 1925}

Material: Russia Far East, Primorskiy Kray, Lazovski Zapovednik, c. $170 \mathrm{~km}$ East of Vladivostok, Ta-Chingousa, $43^{\circ} 01^{\prime} 07^{\prime \prime} \mathrm{N} 134^{\circ} 07^{\prime} 46^{\prime \prime} \mathrm{E}, 0 \mathrm{~m}, 1$ ơ $^{\mathrm{T}} 2001-08-31$ to 2001-09-16, sandy coast, Malaise trap 495, leg. M. Quest (NHM); Russia Far East, Primorskiy Kray, Lazovski Zapovednik, c. $170 \mathrm{~km}$ East of Vladivostok, Korpad, $43^{\circ} 15^{\prime} 52^{\prime \prime} \mathrm{N} 134^{\circ} 07^{\prime} 45^{\prime \prime} \mathrm{E}, 174 \mathrm{~m}, 6$ o $^{\top}$ o $^{\top} 2001-08-05$ to 2001-08-26, sandy flood plain, Malaise trap 489, leg. M. Quest (NHM).

Distribution: Japan and China, new record for Russia Far East.

\section{Coelichneumon haemorrhoidalis (GRAVENHORST, 1820)}

Material: Russia Far East, Primorskiy Kray, Lazovski Zapovednik, c. $170 \mathrm{~km}$ East of Vladivostok, Korpad, $43^{\circ} 17^{\prime} 15^{\prime \prime} \mathrm{N} 134^{\circ} 07^{\prime} 10^{\prime \prime} \mathrm{E}, 526 \mathrm{~m}, 4$ o $^{\text {त }}$ o $^{\top} 2001-06-02$ to 2001-06-25, Malaise trap 466, leg. M. Quest (NHM).

Distribution: West Palaearctic region, new record for the East Palaearctic region.

\section{Coelichneumon octoguttatus UCHIDA, 1925}

Material: Russia Far East, Maritime Province, mouth of Rasdol'naya (= Suyfun) river, 1 क 1974-09-1, leg. V. Zherichin (ZSM); Russia Far East, Maritime Prov., Krasnoarneysk District, Roshchino, 1 ơ 1974-09-21, leg. V. Zherichin (ZSM).

Distribution: Japan, new record for Far East Russia. 


\section{Coelichneumon rufibasalis (UCHIDA, 1927)}

Material: Russia Far East, Primorskiy kray, Anisimovka,

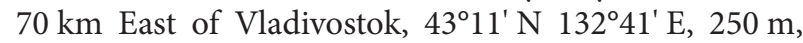
1 o $^{\star 1993-06-7, ~ l e g . ~ A . ~ T a e g e r ~(S D E I) . ~}$

Distribution: Japan and Korea, new record for Far East Russia.

\section{Coelichneumon septenus (Townes, Momol \& Townes, 1965), new o \\ (Fig. 3)}

Material: Russia Far East, Primorskiy kray, Okeanskaya (near Vladivostok), 1 † 1970-09-25, leg. A. Rasnitsyn (ZSM).

Description: 9 . Body length $20 \mathrm{~mm}$. Flagellum with 43 flagellomeres, strongly lanceolate; $1^{\text {st }}$ flagellomere $1.9 \mathrm{x}$ longer than wide, $2^{\text {nd }}$ flagellomere 2.0x longer than wide, combined length $0.5 \mathrm{x}$ eye length; $c .11^{\text {th }}$ flagellomere square; widest flagellomere $2.5 \mathrm{x}$ wider than long. Temple slightly widened behind eye, roundly narrowed apically. Distance of lateral ocellus to eye $1.7 x$ ocellar diameter. Frons rugose-punctate medially, punctate laterally. Face with dense punctures, granulate. Inner eye margins divergent ventrally. Clypeus with coarse punctures, smooth, apical margins slightly concave. Gena wide and swollen, $1.3 \mathrm{x}$ wider than eye, with very scattered punctures. Malar space $0.6 \mathrm{x}$ as long as width of mandibular base.

Mesosoma covered with dense pale brownish hairs. Side of pronotum densely punctate dorsally, striate ventrally and caudally. Notaulus impressed in frontal fourth of mesoscutum. Lateral field of mesoscutum densely punctate, granulate. Mesopleuron densely punctate, speculum smooth. Metapleuron partly rugose-punctate, juxtacoxal carina strong. Scutellum slightly wider than long, with scattered punctures, lateral carina in basal 0.2. Area superomedia about as long as wide, with smooth surface, frontal carina obsolete; costula distinctly caudal to its middle. Area externa punctate, with very fine transversal striae; area petiolaris with lateral carina, rugose. Coxae and femora densely punctate. Hind coxa granulate, with small scopa $(0.2 \mathrm{x}$ coxal length). Hind femur 3.3-3.4x longer than wide; $3^{\text {rd }}$ mid tarsomere c. $1.5 \mathrm{x}$ longer than wide.

Areolet pentagonal, frontal distance of intercubiti 1-2x their width, $2^{\text {nd }}$ recurrent vein distal to its middle, ramellus lacking. Nervulus postfurcal by $1 \mathrm{x}$ its width.

Lateral field of postpetiolus c. $0.7 \mathrm{x}$ as wide as median field, the latter with c. 12 striae. $2^{\text {nd }}$ tergite length c. $0.85 \mathrm{x}$ width. Thyridium transverse, $1.3 \mathrm{x}$ wider than the interval, interval about as wide as the median field of postpetiolus. $2^{\text {nd }}$ to $4^{\text {th }}$ tergites densely punctate, \pm aciculate medially, $5^{\text {th }}$ tergite with very scattered punctures, shining. Ovipositor sheath c. $0.2 \mathrm{x}$ as long as $7^{\text {th }}$ tergite.
Color: Black. Yellowish are flagellomeres 7/8-13/14, wide frontal orbit confluent with vertical spot, small spot on subtegular ridge, apical $2 / 3$ of scutellum, posterolateral spots on $1^{\text {st }}$ to $4^{\text {th }}$ tergites (largest spots on $2^{\text {nd }}$ tergite). Legs black, fore femora apically and fore tibia frontally yellowish; mid tibia paler frontally. Pterostigma chestnut-brown.

Remark: UCHIDA (1935: 14) had described Japanese females from Tosa which he proposed as conspecific with the male holotype of Ichneumon septenguttatus Matsumura (recte: Coelichneumon septenus Townes et al.), but their coloration pattern differ substantially from the type species (e.g. scutellum and $4^{\text {th }}$ tergite without ivory spots). I therefore describe a new female of C. septenus here which corresponds well with the structure and coloration of the male holotype.

Distribution: Japan, new record for Russia Far East.

\section{Coelichneumon sugillatorius (LINNAEUS, 1758)}

Material: Russia Far East, Primorskiy Kray, Lazovski Zapovednik, c. $170 \mathrm{~km}$ East of Vladivostok, Korpad, $43^{\circ} 15^{\prime} 52^{\prime \prime} \mathrm{N} 134^{\circ} 07^{\prime} 45^{\prime \prime} \mathrm{E}, 174 \mathrm{~m}, 1$ o $^{\top} 2001-06-20$ to 2001-06-27, flood plain, Malaise trap 469, leg. M. Quest (NHM); Russia Far East, Primorskiy Kray, Lazovski Zapovednik, c. $170 \mathrm{~km}$ East of Vladivostok, Korpad, $43^{\circ} 17^{\prime} 15^{\prime \prime} \mathrm{N} 134^{\circ} 07^{\prime} 10^{\prime \prime} \mathrm{E}, 526 \mathrm{~m}, 10^{*} 2001-06-02$ to 2001-06-25, Malaise trap 466, leg. M. Quest (NHM).

Distribution: Palaearctic region, known from Russia Far East (RIEDEL, 2012).

\section{Lymantrichneumon disparis (PODA, 1761)}

Material: Russia Far East, Primorskiy Kray, Lazovski Zapovednik, c. $170 \mathrm{~km}$ East of Vladivostok, Korpad, $43^{\circ} 17^{\prime} 16^{\prime \prime} \mathrm{N} 134^{\circ} 07^{\prime} 09^{\prime \prime} \mathrm{E}, 506 \mathrm{~m}, 1$ \& 2001-07-14 to 2001-08-04, Malaise trap 483, coll. M. Quest (NHM); Russia Far East, Primorskiy Kray, Lazovski Zapoved-

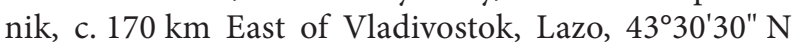
$134^{\circ} 06^{\prime} 58^{\prime \prime} \mathrm{E}, 1353 \mathrm{~m}, 1 \mathrm{o}^{\text {त } 2001-09-07}$ to 2001-09-28, subalpine, Malaise trap 496, leg. M. Quest (NHM).

Distribution: Palaearctic and Oriental regions, known from Far East Russia (Townes et al., 1965).

\section{Protichneumon nigriventris spec. nov. urn:Isid:zoobank.org:act:98F723A9-B2A8-44D8-B118-83F88FAEAE7E (Figs 4, 25, 26, 34)}

Holotype: 우 "USSR, Maritime Prov., Russky I., Aiax Bay, 15.VIII.74, V. Zherichin" (ZSM). 
Description: 우 . Body length $25 \mathrm{~mm}$. Flagellum lanceolate, with 46 flagellomeres; $1^{\text {st }}$ flagellomere $1.8 \mathrm{x}$ longer than wide, $2^{\text {nd }}$ flagellomere $1.4 \mathrm{x}$ longer than wide; widest flagellomere $2.4 \mathrm{x}$ wider than long. Temple slightly narrowed behind eye. Distance of lateral ocellus to eye $1.4 \mathrm{x}$ ocellar diameter. Frons with fine and transversal rugose-striation. Face and clypeus with coarse punctation and fine granulation. Apical margin of clypeus with a wide roundish medial extension. Malar space c. $0.8 \mathrm{x}$ as long as width of mandibular base. Gena $1.1 \mathrm{x}$ wider than eye, with scattered punctures ventrally. Genal carina reaching hypostomal carina far from mandibular base, both carinae low.

Mesosoma covered with short brownish hairs. Pronotum with superficial punctures dorsally and fine longitudinal striation in ventral $2 / 3$. Notaulus distinctly impressed in frontal fifth of mesoscutum. Mesoscutum with dense punctures, finely granulate, but shining. Mesopleuron with coarse punctures, partly striate; speculum smooth and shining. Metapleuron with coarse punctures; juxtacoxal carina present. Scutellum slightly convex, slightly longer than wide, with lateral carina in basal 0.4, with scattered punctures. Propodeum rounded and coarsely rugose. Area superomedia dome-shaped, slightly longer than wide, smooth, distinctly raised above propodeal surface frontally; costula in its middle. Area externa with fine transverse striation. Hind coxa densely punctate, with whitish scopa (length $1 / 3$ of coxal length). Hind femur very stout, c. $2.8 \mathrm{x}$ longer than wide, densely punctate.

Areolet pentagonal, frontal distance of intercubiti $2 \mathrm{x}$ their width. $2^{\text {nd }}$ recurrent vein in its middle. Nervulus slightly postfurcal (1x its width).

Metasoma oxypygous, hypopygium short. Postpetiolus strongly widened; lateral field punctate, $0.7-0.8 \mathrm{x}$ as wide as median field, the latter with fine longitudinal aciculation. Thyridium slightly oblique, $1.2 \mathrm{x}$ wider than their interval. $2^{\text {nd }}$ tergite $1.1 \mathrm{x}$ wider than long. $2^{\text {nd }}$ and $3^{\text {rd }}$ tergites densely punctate, with longitudinal rugose-striation between gastrocoeli. Following tergites with superficial punctures, shining. Ovipositor sheath slightly extending metasomal apex (by $0.4 \mathrm{x}$ length of $7^{\text {th }}$ tergite).

Color: Black. Stripes on flagellomeres 8-14, palps, base of mandible, lateral spots of clypeus, small spot on gena, orbital stripe from upper facial to vertical orbit, collare, subtegular ridge, and apical $2 / 3$ of scutellum yellow. Legs black; apical margins of all trochanters, apical spots on fore and mid femora, subbasal spots on all tibiae and subapical rings of all tarsomeres yellowish. Wings yellowish-hyaline; pterostigma reddish-brown.

Male unknown.

Remark: This new taxon is a typical Protichneumon species, but has an entirely black metasoma which resembles Protichneumon nakanensis (MATsumura, 1912). It differs from P. nakanensis by its black mesoscutum and mesopleuron without yellow spots and mainly black legs with yellow rings of all tibiae and tarsi.

\section{Protichneumon platycerus (KRIECHBAumer, 1895)}

Material: Russia Far East, Primorskiy Kray, Lazovski Zapovednik, c. $170 \mathrm{~km}$ East of Vladivostok, Korpad, $43^{\circ} 17^{\prime} 16^{\prime \prime} \mathrm{N} 134^{\circ} 07^{\prime} 09^{\prime \prime} \mathrm{E}, 506 \mathrm{~m}, 1$ o $^{\star} 2001-07-14$ to 2001-08-04, Malaise trap 483, leg. M. Quest (NHM).

Distribution: East Palaearctic region, known from Russia Far East (UCHIDA, 1941).

\section{Syspasis scutellator (GRAVENHORST, 1829)}

Material: Russia Far East, Khabarovskiy kray, Boitsovo $20 \mathrm{~km}$ North of Bikin, Bolshoi Solutsepyak hill, $300 \mathrm{~m}$, $47^{\circ} 02^{\prime} \mathrm{N} 134^{\circ} 21^{\prime}$ E, 1 ㅇ 1993-05-26, leg. A. Taeger (SDEI).

Distribution: West Palaearctic region including Iran, new record for the East Palaearctic region.

\section{Tribe Ichneumonini}

\section{Achaius oratorius (FABRICIUS, 1793)}

Material: Russia Far East, Primorskiy Kray, Lazovski Zapovednik, c. $170 \mathrm{~km}$ East of Vladivostok, Lazo, 43- ${ }^{\circ} 30^{\prime} 30^{\prime \prime} \mathrm{N} \quad 134^{\circ} 06^{\prime} 58^{\prime \prime} \mathrm{E}, \quad 1353 \mathrm{~m}, 2$ o $^{\top}$ 2001-0907 to 2001-09-28, subalpine, Malaise trap 496, leg. M. Quest (NHM); Primorskiy Kray, Lazovski Zapovednik, c. $170 \mathrm{~km}$ East of Vladivostok, Korpad, $43^{\circ} 17^{\prime} 16^{\prime \prime} \mathrm{N}$ $134^{\circ} 07^{\prime} 09^{\prime \prime} \mathrm{E}, 506 \mathrm{~m}, 1$ ㅇ 2001-07-14 to 2001-08-04, Malaise trap 483, leg. M. Quest (NHM).

Distribution: Palaearctic region, known from Russia Far East (Townes et al., 1965).

\section{Anisopygus pseudonymus WeSMAEL, 1845}

Material: East Siberia, lake Baikal near Anghasolka, $1 \sigma^{*}$ 2007-08-21, leg. O. Berlov (coll. Riedel); Russia Far East, Primorskiy Kray, Lazovski Zapovednik, c. 170 km East of

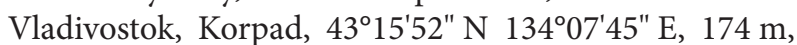
$2 \sigma^{\star} o^{\star}$ 2001-05-30 to 2001-06-06, Malaise trap 453, $2 \sigma^{\star} \sigma^{\star}$ 2001-06-20 to 2001-06-27, flood plain, Malaise trap 469, leg. M. Quest (NHM).

Distribution: Holarctic region, known from Far East Russia (TosQuinet, 1889).

\section{Aoplus defraudator (WeSMAEL, 1845)}

Material: East Siberia, $10 \mathrm{~km}$ East of Irkutsk, $10^{\star}$ 200308-29, leg. O. Berlov (coll. Riedel). 
Distribution: West Palaearctic region, new for the East Palaearctic region.

\section{Aoplus ocellator spec. nov. urn:Isid:zoobank.org:act:48A8BD7B-482E-46DB-A330-DD5EEB39DE4C (Figs 1, 9, 11, 13)}

Holotype: ㅇ "Russia, Primorskiy kray, Tamga, 17 km NE Lesozavodsk, $45.37^{\circ} \mathrm{N} 133.36^{\circ} \mathrm{E}, 24 . \mathrm{V} .1993,130 \mathrm{~m}$, leg. A. Taeger" (SDEI).

Description: ㅇ. Body length $9.5 \mathrm{~mm}$. Flagellum slightly lanceolate, with 32 flagellomeres; $1^{\text {st }}$ flagellomere $3.5 \mathrm{x}$ longer than wide; c. $13^{\text {th }}$ flagellomere square; widest flagellomere c. $1.2 \mathrm{x}$ wider than long. Temple strongly and almost linearly narrowed behind eye, dorsally $0.35 \mathrm{x}$ as long as eye. Ocelli large, distance of lateral ocellus to eye $0.8 \mathrm{x}$ ocellar diameter. Frons granulate and with scattered superficial punctures. Face densely punctate and granulate. Clypeus densely punctate, \pm shining. Gena densely punctate ventrally, $0.7 \mathrm{x}$ as wide as eye. Mandible with two teeth, lower tooth smaller than upper one and slightly bent inwards. Malar space $1.2 \mathrm{x}$ as long as width of mandibular base. Genal carina reaching hypostomal one away from mandibular base, both carinae low.

Mesosoma covered with short brownish hairs. Notaulus impressed in frontal 1/5 of mesoscutum. Mesoscutum granulate and with dense fine punctures, matte. Mesopleuron and metapleuron finely and densely rugose-punctate; speculum punctate, shining. Juxtacoxal carina present. Scutellum moderately elevated, slightly wider than long, with scattered punctures and lateral carina in its basal 0.3 . Areae basalis and superomedia confluent. Area basalis roundly protruding forward medially, but without distinct median tubercle. Area superomedia hexagonal, slightly wider than long; costula distinct medially, obsolete laterally, reaching area superomedia in its middle. Area petiolaris finely rugose, without lateral carina. Hind coxa densely punctate, with large but low whitish scopa. Hind femur densely punctate, 3.9x longer than wide. Claws not pectinate.

Areolet almost quadrangular, frontal distance of intercubiti $1 \mathrm{x}$ their diameter; $2^{\text {nd }}$ recurrent vein in its middle. Nervulus postfurcal (1x its width).

Metasoma oxypygous. Postpetiolus moderately widened, without dorsal carina, finely rugose; lateral field $0.6 \mathrm{x}$ as wide as median field. Gastrocoelus impressed, with some fine ridges. Thyridium large, oblique, $2.0 \mathrm{x}$ wider than the interval. $2^{\text {nd }}$ tergite about as long as wide. $2^{\text {nd }}$ and $3^{\text {rd }}$ tergites granulate and with fine superficial punctures, matte. Following tergites granulate. Ovipositor sheath distinctly extending behind metasomal apex (by length of $3^{\text {rd }}$ hind tarsomere).

Color: Black. Stripes on flagellomeres 7-12 ivory. Scape ventrally, narrow stripe on orbit opposite of antennal grooves, small spot on vertex, stripe on collare and hind edge of pronotum orange. Metasoma black; median field of postpetiolus, $2^{\text {nd }}$ and $3^{\text {rd }}$ tergites completely and $4^{\text {th }}$ tergite basally with dark chestnut-brown shine. Legs black; all femora narrowly red basally; fore and mid tibiae and tarsi brownish frontally; hind tibia dark brownish, infuscate apically. Wings hyaline; pterostigma yellow.

$\sigma^{*}$ : unknown

Remark: This new taxon resembles A. teranishii (UCHIDA, 1929), but differs by a smaller body, distinct scopa on hind coxa, dark reddish-brown basal tergites, yellow pterostigma and black outer orbit.

\section{Chasmias motatorius (FABRICIUS, 1775)}

Material: East Siberia, $10 \mathrm{~km}$ East of Irkutsk, 1 o $^{\star 2} 2007$ 08-09, leg. O. Berlov (coll. Riedel).

Distribution: Palaearctic region, known from West Siberia (WoldstedT, 1881).

\section{Chasmias paludator sibiricus subspec. nov.}

(Figs 2, 10, 12, 14)

Holotype: ㅇ "Russia Far East, Primorskiy kray, Anisimovka (=Kangenus), $70 \mathrm{~km} \mathrm{E} \mathrm{Vladivostok,} 43.11^{\circ} \mathrm{N}$ $132.41^{\circ}$ E, 250 m, 7.VI.1993, leg. A. Taeger" (SDEI).

Paratype: ㅇ "Russia Far East, Khabarovskiy kray, Boitsovo $20 \mathrm{~km} \mathrm{~N}$ Bikin, Kamenistaja Griva Hill, $47.02^{\circ} \mathrm{N}$ 134.25 E, 300 m, 27.V.1993, leg. A. Taeger” (SDEI).

Description: + . Body length $10.5-11 \mathrm{~mm}$. Flagellum filiform, with 27 flagellomeres; $1^{\text {st }}$ flagellomere $2.9 \mathrm{x}$ longer than wide; widest flagellomere c. $1.1 \mathrm{x}$ wider than long. Temple slightly and roundly narrowed behind eye, dorsally $0.75 \mathrm{x}$ as long as eye. Distance of lateral ocellus to eye $1.1 \mathrm{x}$ ocellar diameter. Frons rugose-punctate and weakly granulate. Face transversely rugose-punctate. Clypeus with coarse punctures and coarse longitudinal rugae; apical margin concave, with blunt median tooth. Gena wide, with scattered coarse punctures ventrally. Mandible with two teeth, lower tooth smaller than upper one. Malar space $0.8 \mathrm{x}$ as long as width of mandibular base. Genal carina reaching hypostomal carina away from mandibular base, both carinae low.

Mesosoma covered with short brownish hairs. Notaulus distinctly impressed in frontal $1 / 4$ of mesoscutum. Mesoscutum densely punctate, finely granulate but \pm shining. Mesopleuron and metapleuron densely rugose-punctate, juxtacoxal carina present. Scutellum almost flat, about as long as wide, with scattered fine punctures, without lateral carina. Area superomedia elongate, almost rectangular, c. $1.7 \mathrm{x}$ longer than wide; costula indistinct. Area externa punctate. Area petiolaris rugose, with lateral carina. Hind coxa densely punctate, covered with rather long and dense whitish 
hairs ventrally, but without distinct scopa. Hind femur 3.8-4.1x longer than wide. Claws not pectinate. Areolet pentagonal, frontal distance of intercubiti $2 \mathrm{x}$ their diameter; $2^{\text {nd }}$ recurrent vein slightly distal to its middle. Nervulus postfurcal.

Metasoma oxypygous. Postpetiolus moderately widened, without dorsal carina; lateral field punctate, $0.4 \mathrm{x}$ as wide as median field; median field with few fine striae and punctures, partly smooth and strongly shining. Gastrocoelus impressed, with some fine ridges. Thyridium oblique, $0.7-0.9 \mathrm{x}$ as wide as the interval. $2^{\text {nd }}$ tergite $1.1 \mathrm{x}$ longer than wide. $2^{\text {nd }}$ and $3^{\text {rd }}$ tergites finely and densely rugose-punctate; $2^{\text {nd }}$ tergite with longitudinal rugae in basal half. Ovipositor sheath distinctly extending behind metasomal apex (by length of $2^{\text {nd }}$ hind tarsomere).

Color: Black. Stripes on flagellomeres 7/8-12, scutellum, narrow apical margin of $6^{\text {th }}$ tergite and entire $7^{\text {th }}$ tergite ivory. $2^{\text {nd }}$ tergite with dark brownish suffusion. Legs black; femora narrowly reddish basally; fore and mid tibiae and tarsi \pm reddish; hind tibia reddish in basal $2 / 3$, infuscate apically. Wings hyaline, pterostigma pale ochreous. $o^{\top}$ : unknown.

Remark: This new subspecies differs from the European Chasmias paludator paludator (Desvignes, 1856) by its smaller size, slightly longer basal flagellomeres and mainly blackish instead of red femora.

\section{Cratichneumon jocularis (WESMAEL, 1848)}

Material: East Siberia, $10 \mathrm{~km}$ East of Irkutsk, 1 o $^{\star 2} 2007$ 07-21, leg. O. Berlov (coll. Riedel).

Distribution: Palaearctic region, known from Siberia (Tereshinin, 2003).

\section{Cratichneumon rufifrons (GRAVENHORST, 1829)}

Material: Russia Far East, Primorskiy kray, Anisimovka, Litovka Mts., $70 \mathrm{~km}$ East of Vladivostok, $43^{\circ} 07^{\prime} \mathrm{N}$ $132^{\circ} 41^{\prime}$ E, 900 m, 1 우 1993-06-08, leg. A. Taeger (SDEI); Primorskiy Kray, Lazovski Zapovednik, c. 170 km East of Vladivostok, Korpad, 43ำ $15^{\prime} 52^{\prime \prime} \mathrm{N} 134^{\circ} 07^{\prime} 45^{\prime \prime} \mathrm{E}, 174 \mathrm{~m}$, $10^{\top}$ 2001-06-16 to 2001-06-25, flood plain, Malaise trap 467; $506 \mathrm{~m}, 1$ o 2001-06-20 to 2001-06-27, mountain top, Malaise trap 483; $560 \mathrm{~m}, 1$ o 2001-07-14 to 2001-0804, sandy coast, Malaise trap 498, leg. M. Quest (NHM); Primorskiy Kray, Lazovski Zapovednik, c. 170 km East of Vladivostok, Ta-Chingousa, $43^{\circ} 01^{\prime} 07^{\prime \prime} \mathrm{N} 134^{\circ} 07^{\prime} 46^{\prime \prime} \mathrm{E}$, $0 \mathrm{~m}, 1$ o 2001-08-31 to 2001-09-16, sandy coast, Malaise trap 495, leg. M. Quest (NHM).

Distribution: Palaearctic region, known from Far East Russia (Tereshrin, 2003).

\section{Cratichneumon versator (THUNBERG, 1824)}

Material: Russia Far East, Primorskiy kray, Anisimovka,

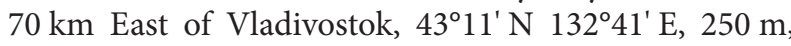
1 ơ 2003-06-07, leg. A. Taeger (SDEI); Russia Far East, Primorskij Kray, Ryazanovska, 14 km Southwest of Slavyanka, $42^{\circ} 48^{\prime} \mathrm{N} 131^{\circ} 12^{\prime} \mathrm{E}, 50 \mathrm{~m}, 1$ क 1993-06-16, leg. Taeger (SDEI); Russia Far East, Ussuriysky Zapovednik, $33 \mathrm{~km}$ Southeast of Ussuriysk, $43^{\circ} 37^{\prime} \mathrm{N} 132^{\circ} 18^{\prime} \mathrm{E}$, 250 m, 2 o o 1993-06-14, leg. A. Taeger (SDEI); Primorskiy Kray, Lazovski Zapovednik, c. $170 \mathrm{~km}$ East of

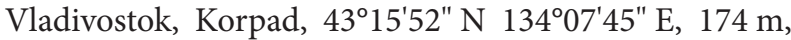
1 o 2001-06-20 to 2001-06-27, flood plain, Malaise trap 469, leg. M. Quest (NHM); Primorskiy Kray, Lazovski Zapovednik, c. $170 \mathrm{~km}$ East of Vladivostok, Korpad, $43^{\circ} 17^{\prime} 16^{\prime \prime} \mathrm{N} 134^{\circ} 07^{\prime} 09^{\prime \prime} \mathrm{E}, 506 \mathrm{~m}, 1$ o $^{*} 2201-06-16$ to 2001-06-25, 1 क 2001-07-14 to 2001-08-04, Malaise trap 483, leg. M. Quest (NHM); Primorskiy Kray, Lazovski Zapovednik, c. $170 \mathrm{~km}$ East of Vladivostok, Ta-Chin-

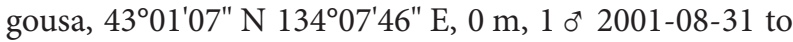
2001-09-16, sandy coast, Malaise trap 495, leg. M. Quest (NHM).

Distribution: Palaearctic region, known from Siberia (Meyer, 1933).

\section{Cratichneumon viator (ScOPOLI, 1763)}

Material: East Siberia, $10 \mathrm{~km}$ East of Irkutsk, $1{ }^{\star}$ 200706-24, leg. O. Berlov (coll. Riedel).

Distribution: Holarctic region, known from Siberia (Meyer, 1933, Tereshinin, 2003).

\section{Ctenichneumon panzeri (WeSMAEL, 1845)}

Material: South Siberia, Southwest Tannu-Ola mountain range, Southwest Tuwa region, Sogly surrounds, $2000 \mathrm{~m}$, 2 우 ㅇ 2003-07-01 to 2003-07-05, leg. Vastchenko (coll. Riedel); Southwest Siberia, Orenburg region, Ilek surrounds, 1 ㅇ, 1 o $^{*}$ 2005-08-01 to 2005-08-05, leg. Osipov (coll. Riedel).

Distribution: Widespread in the Palaearctic and Oriental regions, known from Siberia (MEYER, 1933).

\section{Diphyus akaashii (UCHIDA, 1955)}

Material: Russia, Primorskiy kray, Ryazanovka, 14 km Southwest of Slavyanka, $42^{\circ} 48^{\prime} \mathrm{N} 131^{\circ} 12^{\prime} \mathrm{E}, 50 \mathrm{~m}, 1 \mathrm{o}^{\mathrm{*}}$ 1993-06-16, leg. A. Taeger (SDEI).

Distribution: China, Japan and Korea; new record for Russia Far East. 


\section{Diphyus latebricola (WESMAEL, 1845)}

Material: Russia Far East, Primorskiy kray, Sikhote-Alin, Oblachnaya $56 \mathrm{~km}$ Southeast of Chuguyevka, $43^{\circ} 45^{\prime} \mathrm{N}$ $134^{\circ} 15^{\prime} \mathrm{E}, 850 \mathrm{~m}, 2$ 우 우 1992-06-02, leg. A. Taeger (SDEI); Primorskiy Kray, Lazovski Zapovednik, c. $170 \mathrm{~km}$ East of Vladivostok, Korpad, $43^{\circ} 15^{\prime} 52^{\prime \prime} \mathrm{N}$ $134^{\circ} 07^{\prime} 45^{\prime \prime} \mathrm{E}, 174 \mathrm{~m}, 1$ \% 2001-05-01 to 2001-05-14, flood plain, Malaise trap 441, leg. M. Quest (NHM).

Distribution: West Palaearctic region, new record for the East Palaearctic region.

\section{Eupalamus lamentator (THUNBERG, 1824)}

Material: Russia Far East, Primorskiy Kray, Lazovski Zapovednik, c. $170 \mathrm{~km}$ East of Vladivostok, Korpad, $43^{\circ} 15^{\prime} 52^{\prime \prime} \mathrm{N} \quad 134^{\circ} 07^{\prime} 45^{\prime \prime} \mathrm{E}, 174 \mathrm{~m}, 1$ ㅇ 2001-08-05 to 2001-08-26, sandy flood plain, Malaise trap 489, leg. M. Quest (NHM); Primorskiy Kray, Lazovski Zapovednik, c. $170 \mathrm{~km}$ East of Vladivostok, Korpad, $43^{\circ} 15^{\prime} 52^{\prime \prime} \mathrm{N} 134^{\circ} 07^{\prime} 45^{\prime \prime} \mathrm{E}, 174 \mathrm{~m}, 2$ o $^{\top}$ o $2001-06-20$ to 2001-06-27, flood plain, Malaise trap 469, leg. M. Quest (NHM).

Remark: One $\sigma^{\star}$ with entirely black flagellum, the other $\sigma^{*}$ with an ivory ring on flagellum.

Distribution: Palaearctic region, known from Russia Far East (Meyer, 1933).

\section{Eupalamus oscillator WeSMAEL, 1845}

Material: Russia Far East, Primorskiy Kray, Lazovski Zapovednik, c. $170 \mathrm{~km}$ East of Vladivostok, Korpad, $43^{\circ} 15^{\prime} 52^{\prime \prime} \mathrm{N} 134^{\circ} 07^{\prime} 45^{\prime \prime} \mathrm{E}, 174 \mathrm{~m}, 1$ o $^{\top} 2001-06-20$ to 2001-06-27, flood plain, Malaise trap 469, leg. M. Quest (NHM).

Distribution: Palaearctic region, new record for Russia Far East.

\section{Eutanyacra picta (SCHRANK, 1776)}

Material: Southwest Siberia, Orenburg region, Ilek surrounds, 1 क , $10^{\star}$ 2005-08-01 to 2005-08-05, leg. Osipov (coll. Riedel); Far East Russia, South Sakhalin, $25 \mathrm{~km}$ Northwest of Aniva, 1 o $^{\star}$ 1994-09-01, leg. Osipov (coll. Riedel).

Distribution: Widespread in the Palaearctic and Oriental regions, known from Siberia (MEYER, 1933).

\section{Homotherus magus (WeSMAEL, 1855)}

Material: East Siberia, Lake Baikal, Cape Shartlai, 1 ㅇ 2004-06-20, leg. Berlov (coll. Riedel).

Distribution: Holarctic region, new for Siberia.

\section{Hoplismenus flagellator spec. nov. urn:Isid:zoobank.org:act:675CC203-04A9-4B23-9247-880562A8B0C4 (Figs 18-20)}

Holotype: $\sigma^{*}$ "Far East Russia, S Primorje reg., Shkotovo distr., Anisimovka v., 11-13.VII.2003, leg. Osipov" (coll. Riedel).

Description: $\sigma^{x}$. Body length $10.5 \mathrm{~mm}$. Flagellum with 34 flagellomeres, bristle-shaped; $1^{\text {st }}$ flagellomere $3.4 \mathrm{x}$ longer than wide. Tyloids on flagellomeres 7-14, oval, maximally $0.5 \mathrm{x}$ as long as their flagellomeres and situated slightly basad to center. Temple strongly and almost linearly narrowed behind eye, dorsally $0.75 \mathrm{x}$ as long as eye. Distance of lateral ocellus to eye $0.9 x$ ocellar diameter. Frons coarsely punctate and granulate, matte. Face and clypeus densely punctate and granulate, matte. Clypeus $2.1 \mathrm{x}$ wider than long, apical margin blunt and almost straight. Gena with superficial punctures. Malar space $0.65 \mathrm{x}$ as long as width of mandibular base. Mandible slender, with two teeth, lower tooth smaller than upper one and moderately curved inwards (by c. $45^{\circ}$ ). Genal carina complete, reaching hypostomal carina far from mandibular base, both carinae low.

Mesosoma covered with short grey hairs. Side of pronotum coarsely rugose-punctate dorsally, with coarse rugae ventrally. Notaulus distinctly impressed in frontal 1/6 of mesoscutum. Mesoscutum coarsely and densely punctate and granulate, matte. Mesopleuron and metapleuron coarsely rugose-punctate, speculum finely punctate; juxtacoxal carina present. Scutellum pyramidal-shaped, about as long as wide, densely punctate, with complete lateral carina and fine transverse crest at its top. Propodeum coarsely rugose and completely carinate. Area superomedia hexagonal, $1.5 \mathrm{x}$ wider than long, rugose; costula reaching its middle. Area petiolaris strongly impressed. Outer dentiparal edge with long tooth-like apophysis. Hind femur densely punctate, $5.4 \mathrm{x}$ longer than wide. Claws not pectinate.

Areolet pentagonal, frontal distance of intercubiti $4 \mathrm{x}$ their diameter; $2^{\text {nd }}$ recurrent vein in its middle. Nervulus postfurcal (by $3 \mathrm{x}$ its width).

Postpetiolus moderately widened, lateral field $0.4 \mathrm{x}$ as wide as the median one, punctate; median field elevated, smooth and shining, with coarse punctures. $2^{\text {nd }}$ tergite wide, $1.3 \mathrm{x}$ wider than long. Gastrocoelus rectangularly impressed, with strong ridges; thyridium transverse, c. $0.8 \mathrm{x}$ as wide as the interval. $2^{\text {nd }}$ and $3^{\text {rd }}$ tergites coarsely and densely rugose-punctate, \pm matte. 
Following tergites with superficial punctures, \pm shining. Apical margin of hypopygium with tongue-shaped median extension.

Color: Black. Flagellomeres 9-13, palps, mandible except teeth, labrum, clypeus, entire face and narrow line of frontal orbit, frontal margin and hind edge of pronotum, spot on tegula, subtegular ridge and apical half of scutellum ivory. Coxae and trochanters black; fore and mid coxae with ivory frontal spots; hind trochantellus reddish. Legs otherwise brown; fore and mid femora apically and fore and mid tibiae frontally ivory; hind femur narrowly reddish basally; all tarsi except brown tips of distal tarsomeres and brown basal half of hind metatarsus ivory. Wings hyaline, pterostigma black.

o: unknown.

Remark: This new $\sigma^{\top}$ has entirely black tergites. From

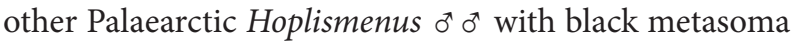
it can be differentiated by the ivory flagellar ring and completely ivory face and clypeus.

\section{Hoplismenus hemimelas HeINRICH, 1978}

Material: Russia Far East, Primorskiy Kray, Lazovski Zapovednik, c. $170 \mathrm{~km}$ East of Vladivostok, Lazo, $43^{\circ} 30^{\prime} 30^{\prime \prime} \mathrm{N} 134^{\circ} 06^{\prime} 58^{\prime \prime} \mathrm{E}, 1353 \mathrm{~m}, 1$ ㅇ 2001-09-07 to 2001-09-28, subalpine, Malaise trap 496, leg. M. Quest (NHM).

Distribution: Only known from Russia Far East (HEINRICH, 1978).

\section{Hoplismenus lamprolabus WeSMAeL, 1857}

Material: South Siberia, Central Tuva region, West Ujuksky mountain range, Kamenuyi river, 1000 m, $1 \sigma^{*}$ 2003-07-11 to 2203-07-20, leg. Vastchenko (coll. Riedel); South Siberia, Altai mountains, Kuralsky mountain range, Aktash village, $2000 \mathrm{~m}, 1$ ㅇ 2003-06-20 to 200306-25, leg. Alexeenko (coll. Riedel).

Distribution: Palaearctic region, known from Siberia (HEINRICH, 1978).

\section{Hoplismenus obscurus KriechBAUMeR, 1895}

Material: Far East Russia, Sikhota-Alin mountains, Ternei environment, 1 o 1985-07-22, leg. Osipov (coll. Riedel).

Distribution: Eastern Palaearctic region, known from Russia Far East (UCHIDA, 1941).

\section{Hoplismenus pica WeSMAEL, 1855}

Material: Far East Russia, Primoskiy kray, Anisimovka,

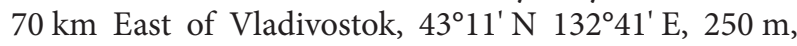
1 ㅇ 1993-06-07, leg. A. Taeger (SDEI).

Distribution: Palaearctic region, known from Russia Far East (HEINRICH, 1978).

\section{Ichneumon brevimaculatus spec. nov. urn:Isid:zoobank.org:act:32A6E211-9DC7-4E78-A7C4-D7C1FD8F1A6C (Figs 21, 27, 35)}

Holotype: of "Russia, Primorskiy kray, Ryazanovka, $14 \mathrm{~km}$ SW Slavyanka, $42.48^{\circ} \mathrm{N} 131.12^{\circ} \mathrm{E}, 50 \mathrm{~m}, 16 . \mathrm{VI}$. 1993, leg. A. Taeger” (SDEI).

Description: 9 . Body length $16 \mathrm{~mm}$. Flagellum bristleshaped, with 41 flagellomeres; $1^{\text {st }}$ flagellomere 2.6x longer than wide, c. $11^{\text {th }}$ flagellomere square, widest flagellomeres about square; preapical flagellomere $1.1 x$ longer than wide. Temple moderately and roundly narrowed behind eye, dorsally $0.8 \mathrm{x}$ as long as eye. Distance of lateral ocellus to eye $1.3 \mathrm{x}$ ocellar diameter. Frons coarsely rugose-punctate, with a median blunt crest-like elevation, smooth above antennal sockets. Face densely rugose-punctate and granulate, matte. Clypeus densely punctate, \pm shining, apical margin slightly concave. Gena $1.2 \mathrm{x}$ wider than eye, densely punctate. Malar space $1.2 \mathrm{x}$ longer than width of mandibular base. Mandible with two teeth, lower tooth smaller than upper one. Genal carina complete, reaching hypostomal carina away from mandibular base, both carinae low.

Mesosoma covered with brownish hairs. Notaulus distinctly impressed in frontal $1 / 5$ of mesoscutum. Mesoscutum densely punctate and granulate, matte. Mesopleuron and metapleuron densely rugose-punctate; juxtacoxal carina present. Scutellum moderately elevated, $1.2 \mathrm{x}$ wider than long, with scattered punctures and without lateral carina. Propodeum coarsely rugose. Area superomedia slightly hexagonal, 1.1x wider than long, rugose; costula weakly developed. Area petiolaris transversely rugose, with lateral carinae. Outer dentiparal edge with short blunt tooth-like extension. Hind coxa with dense punctures, without scopa. Hind femur densely punctate, $4.0 \mathrm{x}$ longer than wide.

Areolet pentagonal, frontal distance of intercubiti $2 x$ their diameter; $2^{\text {nd }}$ recurrent vein distal to its middle. Nervulus slightly postfurcal ( $1 \mathrm{x}$ its width).

Metasoma strongly oxypygous, hypopygium short. Postpetiolus strongly widened, lateral field $0.8 \mathrm{x}$ as wide as the median one, punctate; median field largely smooth and shining, with some fine longitudinal striae and scattered punctures. $2^{\text {nd }}$ tergite very wide, $1.35 \mathrm{x}$ wider than long. Gastrocoelus impressed, with strong ridges; thyridium almost transverse, c. $0.7 \mathrm{x}$ as wide as the interval. $2^{\text {nd }}$ and 
$3^{\text {rd }}$ tergites very finely and densely rugose-punctate, \pm matte. Following tergites granulate, slightly shining. $2^{\text {nd }}$ to $4^{\text {th }}$ sternites with median folds. Ovipositor sheath slightly extending behind metasomal apex.

Color: Black. Flagellomeres 8-12 with yellow stripes. Scutellum, triangular posterolateral spots on $1^{\text {st }}$ and $2^{\text {nd }}$ tergites and large median spots on $4^{\text {th }}$ to $7^{\text {th }}$ tergites yellow. Coxae and trochanters black; legs including trochantelli otherwise reddish. Wings with slightly yellowish tint; pterostigma yellow.

$o^{*}$ : unknown.

Remark: This new species belongs to group D sensu HilPERT (1992). In the key of Siberian Ichneumon species (RIEDEL, 2018), it runs to couplet 22: Ichneumon quaesitorius LinNAEus, but differs by the yellow spots on $1^{\text {st }}$ and $2^{\text {nd }}$ tergites and the completely red hind tibia and tarsus. The taxon resembles Ichneumon sexmaculatus Matsumura, 1912, but differs by the large yellow spot on $4^{\text {th }}$ tergite and reddish legs.

Ichneumon flavocoxalis spec. nov.

urn:Isid:zoobank.org:act: F0B7571F-F53D-4F5E-AEF7-E8E0DEC72F1D

(Figs 5, 22, 28)

Holotype: † "Russia, Primorskij Kray, Ryazanovska, $14 \mathrm{~km}$ SW Slavyanka, $42^{\circ} 48^{\prime} \mathrm{N} 131^{\circ} 12^{\prime} \mathrm{E}, 50 \mathrm{~m}, 16$.VI. 1993, leg. A. Taeger" (SDEI).

Description: + . Body length $19 \mathrm{~mm}$. Flagellum bristleshaped, with 44 flagellomeres; $1^{\text {st }}$ flagellomere $2.4 \mathrm{x}$ longer than wide, $8^{\text {th }}$ flagellomere square, widest flagellomeres $1.2 \mathrm{x}$ wider than long; preapical flagellomere $1.2 \mathrm{x}$ longer than wide. Temple moderately and roundly narrowed behind eye, dorsally $0.8 \mathrm{x}$ as long as eye. Distance of lateral ocellus to eye $1.3 \mathrm{x}$ ocellar diameter. Frons coarsely rugose-punctate. Face densely rugose-punctate and granulate, matte. Clypeus densely rugose-punctate, \pm shining. Gena $1.3 x$ wider than eye, densely rugose-punctate. Malar space $1.1 \mathrm{x}$ longer than width of mandibular base. Mandible with two teeth, lower tooth smaller than upper one. Genal carina complete, reaching hypostomal carina away from mandibular base; both carinae low.

Mesosoma covered with brownish hairs. Notaulus indistinctly impressed. Mesoscutum densely and finely rugose-punctate, matte. Mesopleuron and metapleuron densely rugose-punctate; juxtacoxal carina present. Scutellum moderately elevated, slightly wider than long, with dense punctures, without lateral carina. Propodeum completely carinate. Area superomedia hexagonal, $1.1 \mathrm{x}$ wider than long, rugose; costula reaching area superomedia slightly frontal to its middle, costula weak laterally. Area externa and area petiolaris rugose. Outer dentiparal edge with short blunt tooth. Hind coxa with fine dense punctures, without scopa. Hind femur densely punctate, $4.0 x$ longer than wide. $3^{\text {rd }}$ mid tarsomere c. $1.6 \mathrm{x}$ longer than wide.
Areolet pentagonal, frontal distance of intercubiti $3 x$ their diameter; $2^{\text {nd }}$ recurrent vein slightly distal to its middle. Nervulus slightly postfurcal ( $1 \mathrm{x}$ its width).

Metasoma strongly oxypygous, hypopygium short. Postpetiolus strongly widened, lateral field $0.9 \mathrm{x}$ as wide as median field, with coarse punctures; median field with very fine and superficial aciculation. $2^{\text {nd }}$ tergite very wide, $1.35 \mathrm{x}$ wider than long. Gastrocoelus impressed, with weak ridges; thyridium slightly oblique, c. $0.65 \mathrm{x}$ as wide as the interval. $2^{\text {nd }}$ and $3^{\text {rd }}$ tergites very finely and densely rugose-punctate, \pm matte. Following tergites granulate, slightly shining. $2^{\text {nd }}$ to $4^{\text {th }}$ sternites with median folds. Ovipositor sheath slightly extending behind metasomal apex.

Color: Black. Flagellomeres 5-13 with ivory stripes. Collare and upper margin of pronotum, spot on tegula, subtegular ridge, scutellum, posterolateral spots on $1^{\text {st }}$ to $3^{\text {rd }}$ tergites (smaller and weak on $3^{\text {rd }}$ tergite) and large median spots on $5^{\text {th }}$ to $7^{\text {th }}$ tergites yellow. Coxae and trochanters black; all coxae with dorsal yellow spots; hind trochantellus dark reddish. Legs otherwise red; fore and mid femora with blackish ventral suffusion; hind femur narrowly black apically; all tibiae with subbasal yellow rings or stripes; hind tibiae infuscate in apical 0.2 ; hind tarsus black. Wings with yellowish tint; pterostigma yellow.

$o^{\star}$ : unknown.

Remark: This large species belongs to group B sensu Hilpert (1992), but differs from all known species in this group by its black basal tergites with posterolateral yellow spots. In the key of Siberian Ichneumon species (RIEDEL, 2018) it runs to couplet 6: Ichneumon quaesitorius LiNNAEUs, but it can be differentiated by the yellow spots on hind coxa and basal tergites and the subbasal yellow bands of all tibiae.

\section{Ichneumon flavotibialis spec. nov. urn:Isid:zoobank.org:act:07E8B9DD-3819-4F13-B988-5FAC6CBA25C3 (Figs 24, 32, 39)}

Holotype: o "Russia, Primorskiy kray, Anisimovka (= Kangaus), $70 \mathrm{~km}$ East of Vladivostok, $43^{\circ} 11^{\prime} \mathrm{N}$ $132^{\circ} 41^{\prime}$ E, 250 m, 07.VI.1993, leg. A. Taeger” (SDEI).

Description: 우 . Body length $14.5 \mathrm{~mm}$. Flagellum almost bristle-shaped, with 44 flagellomeres; $1^{\text {st }}$ flagellomere $2.0 \mathrm{x}$ longer than wide, $9^{\text {th }}$ flagellomere square, widest flagellomeres $1.3 \mathrm{x}$ wider than long; preapical flagellomere about as long as wide. Temple strongly and roundly narrowed behind eye, dorsally $0.75 \mathrm{x}$ as long as eye. Distance of lateral ocellus to eye $1.3 x$ ocellar diameter. Frons coarsely rugose-punctate. Face densely and transversely rugosepunctate and granulate, matte. Clypeus with scattered punctures, \pm shining. Gena as wide as eye, with rather dense punctures. Malar space $1.1 \mathrm{x}$ longer than width of mandibular base. Mandible with two teeth, lower 
tooth smaller than upper one. Genal carina complete, reaching hypostomal carina away from mandibular base; hypostomal carina slightly elevated.

Mesosoma covered with short brownish hairs. Notaulus impressed in frontal 1/6 of mesoscutum. Mesoscutum coarsely rugose-punctate, matte. Mesopleuron densely punctate, partly striate ventrally; speculum punctate. Metapleuron coarsely rugose; juxtacoxal carina present. Scutellum moderately elevated, $1.2 \mathrm{x}$ wider than long, with fine punctures, without lateral carina. Propodeum completely carinate. Area superomedia hexagonal, about as wide as long, rugose; costula reaching area superomedia in its middle, weak laterally. Area petiolaris with transverse rugae, lateral carina present. Outer dentiparal edge without tooth. Hind coxa with dense punctures, with small and slightly elevated scopa apico-ventrally. Hind femur with scattered punctures in ventral half, $4.1 \mathrm{x}$ longer than wide. Tarsomeres not widened, $3^{\text {rd }} \mathrm{mid}$ tarsomere c. 1.6x longer than wide.

Areolet pentagonal, frontal distance of intercubiti $3-4 \mathrm{x}$ their diameter; $2^{\text {nd }}$ recurrent vein distal to its middle. Nervulus postfurcal by $2 \mathrm{x}$ its width.

Metasoma semi-amblypygous, hypopygium moderately elongate, about as long as the distance of its apical margin to metasomal apex. Postpetiolus strongly widened, lateral field $0.6 \mathrm{x}$ as wide as median field, with few striae; median field with fine regular striation. $2^{\text {nd }}$ tergite very wide, $1.35 \mathrm{x}$ wider than long. Gastrocoelus slightly impressed, almost smooth; thyridium narrow, slightly oblique, c. $0.7 \mathrm{x}$ as wide as the interval. $2^{\text {nd }}$ and $3^{\text {rd }}$ tergites very finely and densely rugose-punctate, \pm matte; $2^{\text {nd }}$ tergite finely striate between thyridia. Following tergites finely punctate, shining. $2^{\text {nd }}$ to $4^{\text {th }}$ sternites with median folds. Ovipositor sheath not extending behind metasomal apex.

Color: Black. Flagellomeres 9-16, palps, mandible except teeth, clypeus, face except black median stripe, frontal orbit, ventral spot on scape, collare and wide upper margin of pronotum, two short paramedian stripes on mesoscutum, subtegular ridge, scutellum, fore and mid coxae, all trochanters, trochantelli, tibiae and tarsi yellowish. Femora blackish; fore and mid femora with frontal yellow stripes; hind femur narrowly yellowish apically. $2^{\text {nd }}$ tergite reddish-yellow, with wide triangular apical spot; $3^{\text {rd }}$ tergite black, with lateral reddish-yellow stripe; $2^{\text {nd }}$ and $3^{\text {rd }}$ sternites entirely and fold of $4^{\text {th }}$ sternite yellowish. Wings with slightly yellowish tint; pterostigma yellow.

$o^{\top}:$ unknown.

Remark: Due its small and slightly elevated scopa on hind coxa, this new taxon belongs to the group E1 sensu Hilpert (1992). It differs from all known Palaearctic species of this group by a combination of semi-amblypygous metasoma, yellow paramedian spots on mesoscutum, absence of pale spots on apical tergites, and extended yellow coloration of legs.

\section{Ichneumon haglundi HOLmgren, 1864}

Material: Far East, Primorskij Kray, Ryazanovska, 14 km Southwest of Slavyanka, $42^{\circ} 48^{\prime} \mathrm{N} 131^{\circ} 12^{\prime} \mathrm{E}, 50 \mathrm{~m}, 1$ 우 1993-06-16, leg. Taeger (SDEI).

Distribution: Palaearctic region, known from Siberia (RIEDEL, 2018).

\section{Ichneumon magistratus HILPERT, 1992}

Material: Russia Far East, Primorskiy kray, Przhevalski Mountains, $53 \mathrm{~km}$ Southeast of Ussuriysk, $43^{\circ} 37^{\prime} \mathrm{N}$ $132^{\circ} 35^{\prime}$ E, 250 m, 1 ㅇ 1993-06-13, leg. A. Taeger (SDEI).

Distribution: Known from Russia Far East (HILPeRT, 1992).

\section{Ichneumon megextensorius spec. nov.} urn:Isid:zoobank.org:act:3F921DA4-7285-4328-85AB-C32096485FA8 (Figs 6, 23, 30, 37)

Holotype: \& "Russia Khabarovskiy kray, Boitsovo $20 \mathrm{~km} \mathrm{~N}$ Bikin, Kamenistaja Griva Hill, $47.02^{\circ} \mathrm{N}$ 134.25 E, 300 m, 27.V.1993, leg. A. Taeger” (SDEI).

Description: 우 . Body length $16.3 \mathrm{~mm}$. Flagellum slightly lanceolate, with 44 flagellomeres; $1^{\text {st }}$ flagellomere $1.85 \mathrm{x}$ longer than wide, c. $7^{\text {th }}$ flagellomere square, widest flagellomeres $1.8 \mathrm{x}$ wider than long; preapical flagellomere $0.7 \mathrm{x}$ as long as wide. Temple moderately and roundly narrowed behind eye, dorsally $0.75 \mathrm{x}$ as long as eye. Distance of lateral ocellus to eye $1.2 \mathrm{x}$ ocellar diameter. Frons coarsely rugose-punctate. Face densely punctate and shining. Clypeus densely punctate, shining; apical margin straight. Gena 1.2x wider than eye, with scattered punctures ventrally. Malar space as long as width of mandibular base. Mandible with two teeth, lower tooth smaller than upper one. Genal carina complete, reaching hypostomal carina away from mandibular base, both carinae low.

Mesosoma covered with pale greyish hairs. Notaulus distinctly impressed in frontal 1/6 of mesoscutum. Mesoscutum densely rugose-punctate, shining. Mesopleuron densely punctate, partly striate. Metapleuron coarsely rugose-punctate; juxtacoxal carina indistinct. Scutellum almost flat, $1.1 \mathrm{x}$ wider than long, with scattered punctures, without lateral carina. Propodeum coarsely rugose. Area superomedia almost rectangular, $1.4 \mathrm{x}$ longer than wide, rugose; costula absent. Area petiolaris rugose, with lateral carinae. Outer dentiparal edge without toothlike lamella. Hind coxa with dense punctures and large greyish scopa (length $1 / 3$ of coxal length). Hind femur with scattered punctures in ventral $1 / 3,3.6 \mathrm{x}$ longer than wide. Hind tibia with few external spurs. 

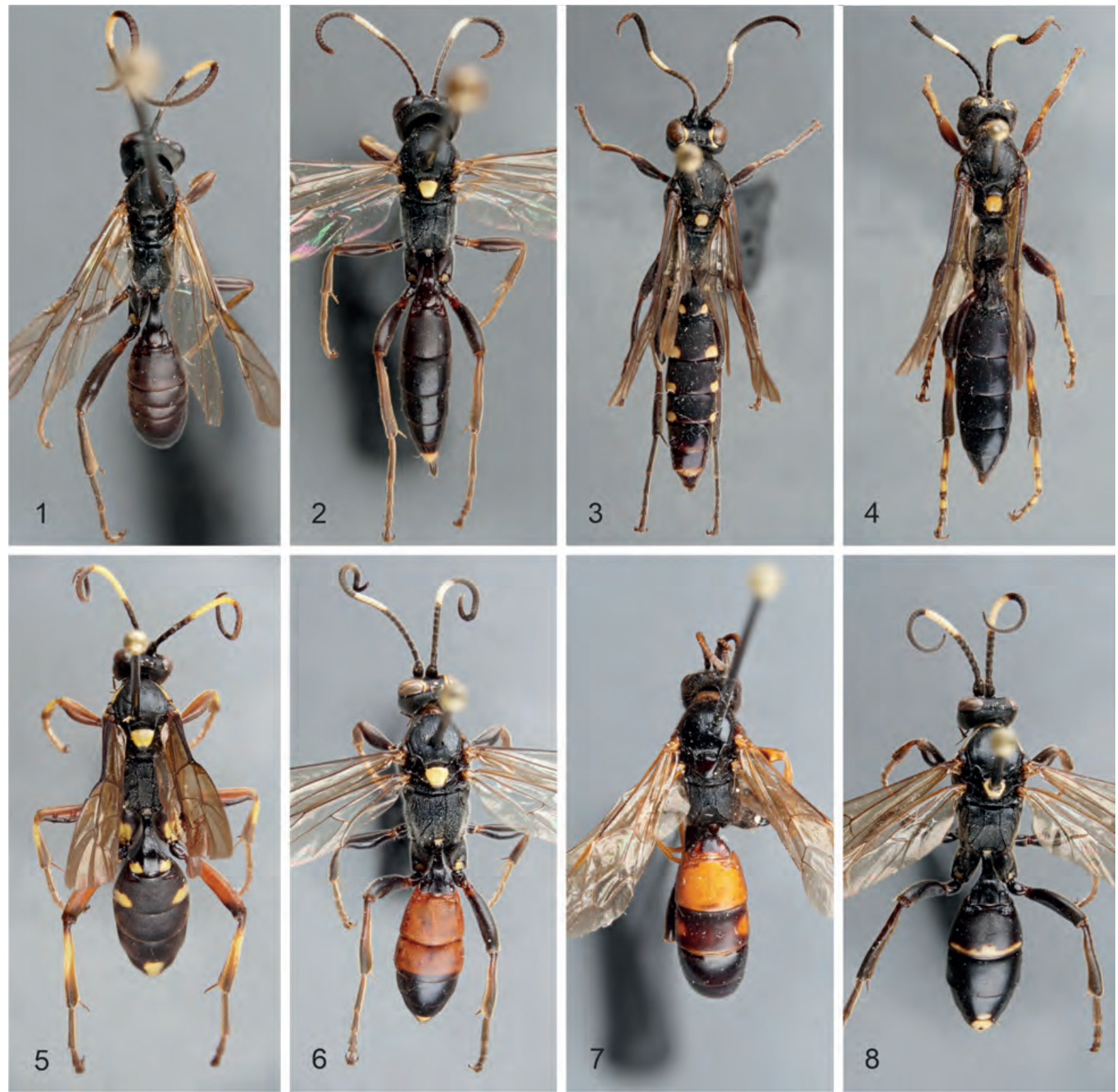

Figs 1-8: Habitus of: 1. Aoplus ocellator spec. nov. ㅇ, 2. Chasmias paludator sibiricus subspec. nov., 3. Coelichneumon septenus Townes et al., new 우, 4. Protichneumon nigriventris spec. nov. 우 5. Ichneumon flavocoxalis spec. nov. ㅇ, 6. Ichneumon megextensorius spec. nov., 7. Ichneumon pilocephalus spec. nov., 우, 8. Ichneumon taegeri spec. nov. 우

Areolet pentagonal, frontal distance of intercubiti $3 x$ their diameter; $2^{\text {nd }}$ recurrent vein distal to its middle. Nervulus postfurcal ( $2 \mathrm{x}$ its width).

Metasoma strongly oxypygous, hypopygium short. Postpetiolus strongly widened, lateral field $0.5 \mathrm{x}$ as wide as the median one; postpetiolus with regular fine aciculation. $2^{\text {nd }}$ tergite $1.2 \mathrm{x}$ wider than long. Gastrocoelus impressed, but widely smooth, with few short ridges; thyridium almost transverse, c. $0.8 \mathrm{x}$ as wide as the interval. $2^{\text {nd }}$ and $3^{\text {rd }}$ tergites finely and densely rugosepunctate, \pm matte; $2^{\text {nd }}$ tergite with median longitudinal rugae in basal half. Following tergites granulate, shining. $2^{\text {nd }}$ to $3^{\text {rd }}$ sternites with median folds. Ovipositor sheath slightly extending behind metasomal apex.
Color: Black. Stripes of flagellomeres 8-14, scutellum and large median spots on $6^{\text {th }}$ and $7^{\text {th }}$ tergites ivory. Frontal orbit with narrow yellowish stripe (widened dorsally). Collare, hind edge of pronotum and tegula reddish. Small spot on subtegular ridge yellowish. $2^{\text {nd }}$ and $3^{\text {rd }}$ tergites red; $3^{\text {rd }}$ tergite with black band in apical 0.2. Legs black; fore and mid tibiae reddish frontally; hind trochantellus and narrow base of hind femur reddish; hind tibia reddish, black in apical 0.25; hind tarsus except red proximal half of metatarsus black. Wings with slight yellowish tint; pterostigma reddish.

$o^{*}$ : unknown.

Remark: This new species belongs to group E2 sensu HILPERT (1992). In the key of Siberian Ichneumon species 

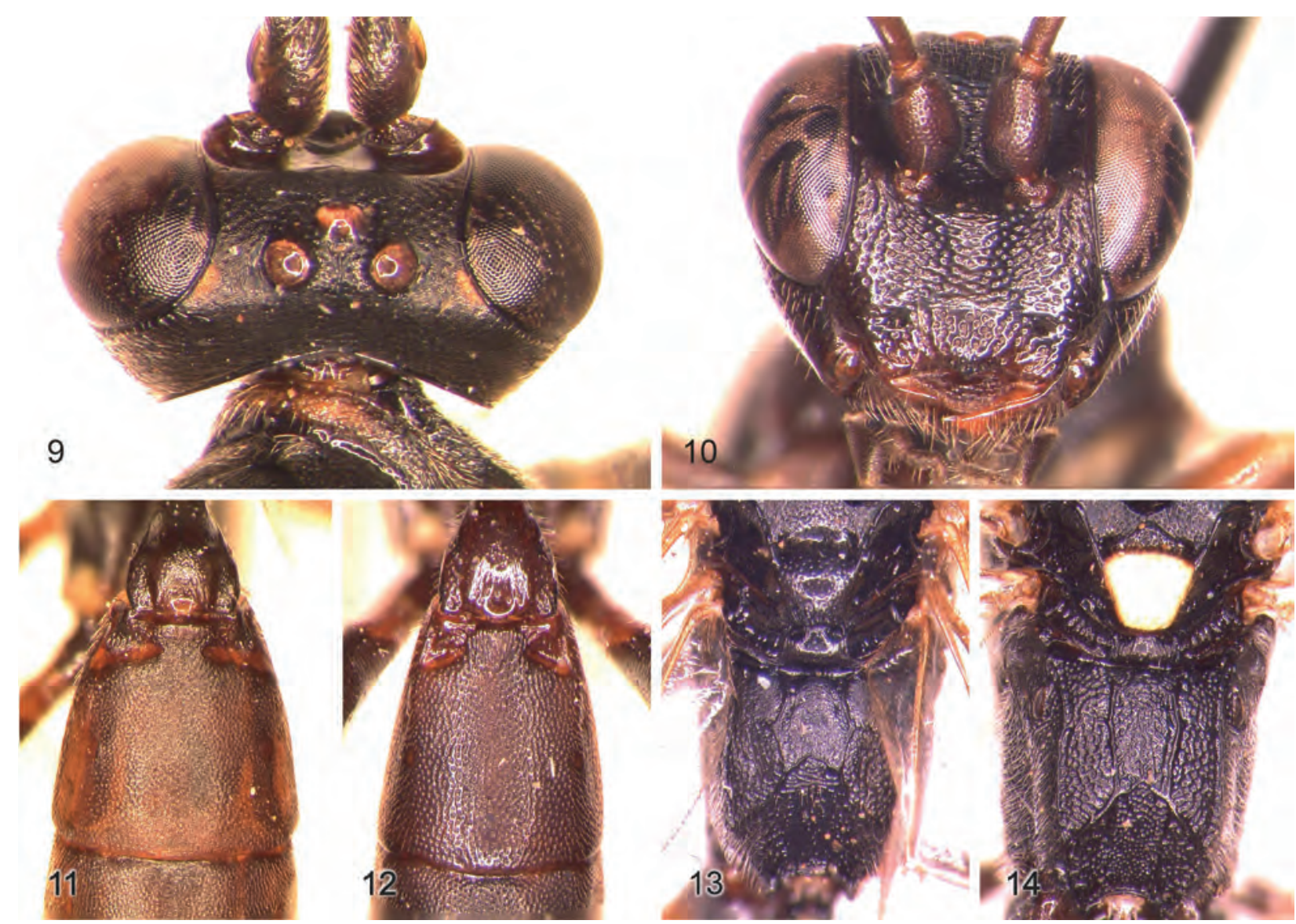

Fig. 9: Head from dorsal of Aoplus ocellator spec. nov. ㅇ, 10. Face of Chasmias paludator sibiricus subspec. nov. ․ . - Figs 11-12: Basal tergites of: 11. Aoplus ocellator spec. nov. ㅇ, 12. Chasmias paludator sibiricus subspec. nov. ․ - Figs 13-14: Propodeum of: 13. Aoplus ocellator spec. nov. ㅇ, 14. Chasmias paludator sibiricus subspec. nov. ㅇ.

(RIEDEL, 2018) it runs to couplet 43: Ichneumon extensorius Linnaeus, but differs by its larger size, longer flagellum with 44 flagellomeres, longer area superomedia, and black apical band of $3^{\text {rd }}$ tergite.

\section{Ichneumon pilocephalus spec. nov.}

urn:Isid:zoobank.org:act:FCB03748-C2BF-47B5-9439-397B6E944973

(Figs $7,31,38,43,44$ )

Holotype: ㅇ “21.07.1991, Mutnovaki-Vulkan, SüdKamtschatka, leg. R. Predel” (Erfurt).

Description: + . Body length $13 \mathrm{~mm}$. Flagellum almost filiform, with 40 flagellomeres; $1^{\text {st }}$ flagellomere $1.5 \mathrm{x}$ longer than wide, c. $6^{\text {th }}$ flagellomere square, widest flagellomeres $1.4 \mathrm{x}$ wider than long; preapical flagellomere $0.75 \mathrm{x}$ as long as wide.

Head covered with rather long and erect brown hairs. Temple moderately and roundly narrowed behind eye, dorsally $0.75 \mathrm{x}$ as long as eye. Distance of lateral ocellus to eye $1.7 x$ ocellar diameter. Frons densely punctate. Face transversely rugose-punctate. Clypeus slightly convex and with weak medio-apical impression, with scattered punctures. Gena $1.3 \mathrm{x}$ wider than eye, with scattered punctures ventrally. Malar space 1.0x longer than width of mandib- ular base. Mandible slender and long, upper tooth with rounded tip, lower tooth very small and only indicated as small extension on lower margin of upper tooth. Genal carina complete, reaching hypostomal carina away from mandibular base, both carinae low.

Mesosoma covered with erect brownish hairs. Side of pronotum largely striate, punctate on upper margin. Notaulus not impressed. Mesoscutum densely punctate frontally, with scattered fine punctures in apical half. Mesopleuron and metapleuron densely striate-punctate; juxtacoxal carina present. Scutellum moderately elevated, about as long as wide, with fine scattered punctures and without lateral carina. Propodeum rugose. Area superomedia square, finely rugose; costula absent. Area petiolaris rugose, without lateral carinae. Hind coxa with dense brown hairs and dense punctures, with blunt longitudinal edge ventrally, without scopa. Hind femur with scattered punctures in ventral half, $3.8 x$ longer than wide. Hind tibia with c. 6 apico-external spurs. Tarsomeres not widened.

Areolet pentagonal, frontal distance of intercubiti $4 \mathrm{x}$ their diameter; $2^{\text {nd }}$ recurrent vein in its middle. Nervulus slightly postfurcal by $1 \mathrm{x}$ its width.

Metasoma strongly oxypygous, hypopygium short. Postpetiolus strongly widened, lateral field $0.5 \mathrm{x}$ as wide as the median one, punctate; median field with fine regular 

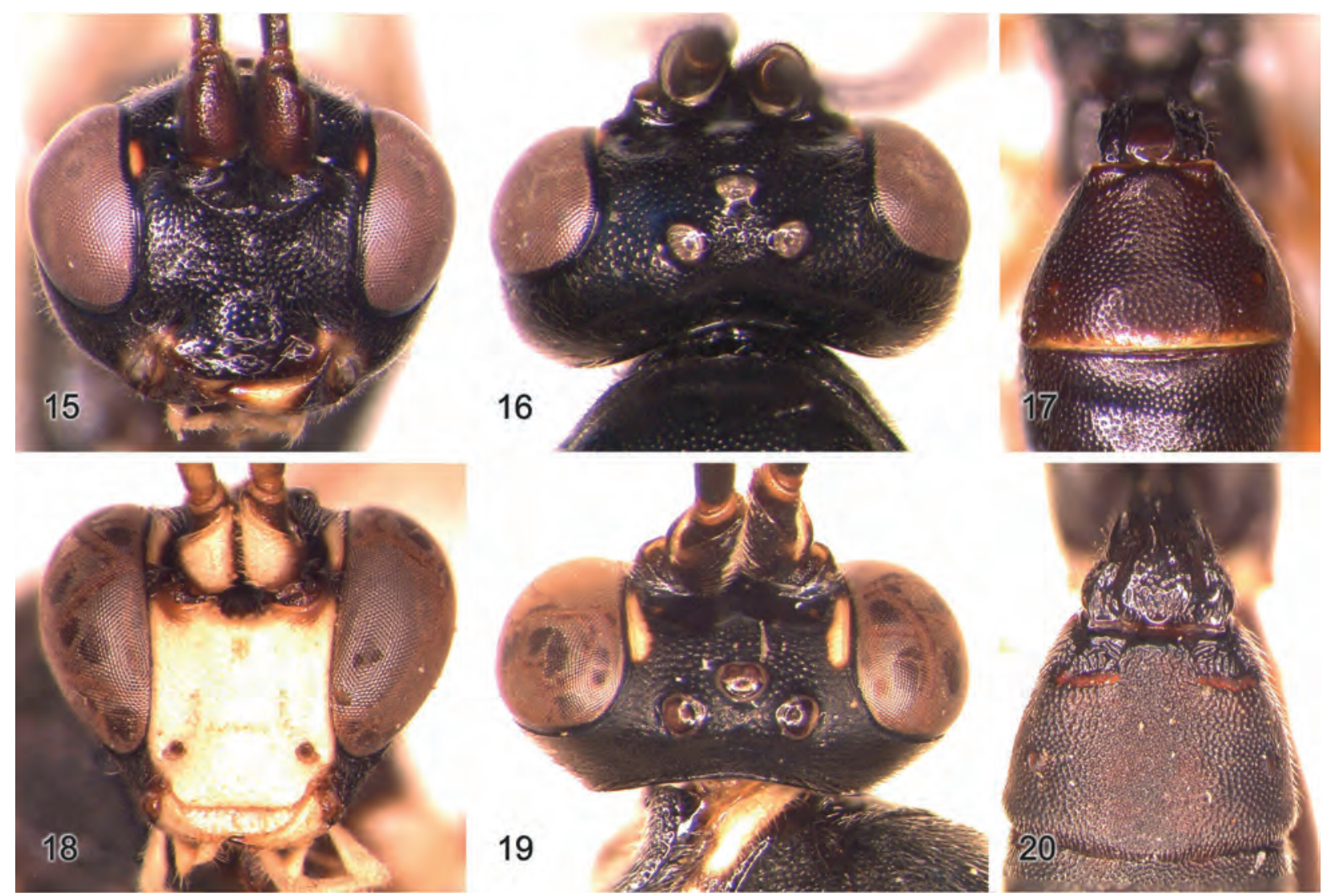

Figs 15-17: Ectopius sibiricus spec. nov. + : 15. face, 16. head from dorsal, 17. basal tergites. - Figs 18-20: Hoplismenus flagellator spec. nov. $\sigma^{\top}$ : 18. face, 19. head from dorsal, 20. basal tergites.

striation. $2^{\text {nd }}$ tergite $1.3 \mathrm{x}$ wider than long. Gastrocoelus impressed, with strong ridges; thyridium almost transverse, c. $0.75 \mathrm{x}$ as wide as the interval. $2^{\text {nd }}$ and $3^{\text {rd }}$ tergites densely punctate, without median rugae. $4^{\text {th }}$ tergite with fine dense punctures, following tergites with very superficial punctures. $2^{\text {nd }}$ to $4^{\text {th }}$ sternites with median folds. Ovipositor sheath slightly extending behind metasomal apex.

Color: Black. Flagellum black, central flagellomeres (c. 8-13) \pm reddish ventrally, but flagellum without clear pale ring. Palps, mandible centrally and median spot on face reddish. Collare and tegula red; scutellum with dark reddish shine. Postpetiolus apically, $2^{\text {nd }}$ tergite entirely and lateral spots on $3^{\text {rd }}$ tergite red. $7^{\text {th }}$ tergite with median ivory spot. Coxae and trochanters black; hind trochantellus red; mid femur except red apex and hind femur completely black. Tibiae and tarsi red; hind tibia with weak infuscation in apical 0.1 . Wings slightly yellowish; pterostigma yellow-red.

$o^{\star}$ : unknown.

Remark: Due to the ventral edge of hind coxa, this new species belongs to the group E4 sensu Hilpert (1992). It is characterized by the erect pilosity on head and mesosoma, modified form of mandible, and absence of pale flagellar ring.

In the key for Siberian Ichneumon (RIEDEL, 2018), this new taxon runs to couplet 46 (Ichneumon mandibulatus
RIEDEL). It differs from I. mandibulatus by the slenderer mandible, erect pilosity, mainly black $3^{\text {rd }}$ tergite, absence of ivory spots on $5^{\text {th }}$ and $6^{\text {th }}$ tergites, and dark reddish scutellum.

Ichneumon polyxanthus (KRIECHBAUMER, 1869)

(Fig. 29, 36)

Material: Russia, Khabarovskiy kray, Boitsovo $20 \mathrm{~km}$ North of Bikin, Bolshoi Solutsepyok hill, $47.02^{\circ} \mathrm{N}$ $134.21^{\circ}$ E, 1 ㅇ 1993-05-26, 300 m, leg. A. Taeger (SDEI).

Distribution: Europe, new record for the East Palaearctic region.

\section{Ichneumon submarginatus GRAVENHORST, 1829}

Material: Russia Far East, Primorskiy kray, Anisimovka, $70 \mathrm{~km}$ East of Vladivostok, $43.11^{\circ} \mathrm{N} 132.41^{\circ} \mathrm{E}, 250 \mathrm{~m}, 1$ 우 1993-06-07, leg. A. Taeger (SDEI); Russia, Khabarovskiy kray, Boitsovo $20 \mathrm{~km}$ North of Bikin, Kamenistaja Griva Hill, $47.02^{\circ} \mathrm{N} 134.25^{\circ} \mathrm{E}, 300 \mathrm{~m}, 1$ \% 1993-05-26, leg. A. Taeger (SDEI).

Distribution: Palaearctic region, known from Far East Russia (Meyer, 1933). 

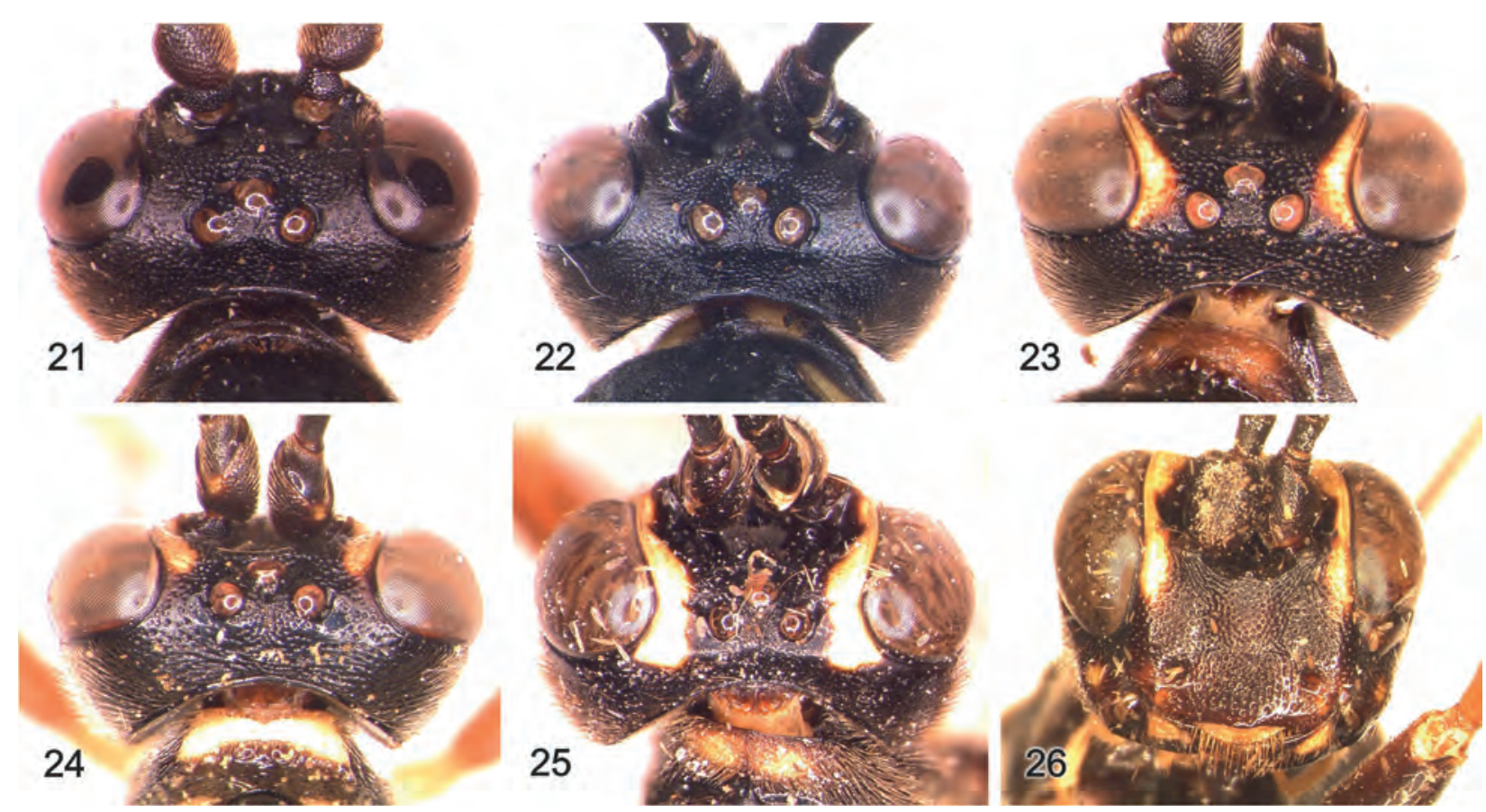

Figs 21-23: Head from dorsal: 21. Ichneumon brevimaculatus spec. nov. $+: 22$. Ichneumon flavocoxalis spec. nov. + , 23. Ichneumon megextensorius spec. nov. + , 24. Ichneumon flavotibialis spec. nov. - Figs 25-26: Protichneumon nigriventris spec. nov. $9: 25$. Head from dorsal, 26. face.

\section{Ichneumon taegeri spec. nov.}

urn:Isid:zoobank.org:act:2EFB6CDD-10E9-4D81-8639-D8E54F68DC08 (Fig. 8)

Holotype: ㅇ "Russia, Primorskiy kray, Smarka $70 \mathrm{~km} \mathrm{~N}$ Chuguyevka, 44.43 N 134.12 E, 30.V.1993, $200 \mathrm{~m}$, leg. A. Taeger" (SDEI).

Description: + . Body length $14.5 \mathrm{~mm}$. Flagellum slightly lanceolate, with 40 flagellomeres; $1^{\text {st }}$ flagellomere $1.6 \mathrm{x}$ longer than wide, $3^{\text {rd }}$ or $4^{\text {th }}$ flagellomere square, widest flagellomeres $1.5 \mathrm{x}$ wider than long; preapical flagellomere $0.9 \mathrm{x}$ as long as wide. Temple distinctly and almost linearly narrowed behind eye. Distance of lateral ocellus to eye $1.5 x$ ocellar diameter. Frons and face transversely rugose-punctate. Clypeus with scattered punctures, intervals smooth and shining. Gena $1.1 x$ wider than eye, with scattered punctures. Malar space as long as width of mandibular base. Mandible with two teeth, lower tooth smaller than upper one. Genal carina complete, reaching hypostomal carina away from mandibular base, both carinae low.

Mesosoma covered with brownish hairs. Notaulus shortly impressed at frontal margin of mesoscutum. Mesoscutum densely punctate, shining. Mesopleuron and metapleuron densely punctate, partly rugose; juxtacoxal carina present. Scutellum almost flat, about as long as wide, with dense punctures, without lateral carina. Propodeum completely carinate. Area superomedia square, rugose; costula absent. Area externa and area petiolaris rugose, area petiolaris without lateral carina. Outer dentiparal edge without tooth. Hind coxa with fine dense punctures, without scopa or crest. Hind femur with scattered punctures in ventral $1 / 4,3.4 \mathrm{x}$ longer than wide. Tarsomeres slightly widened; $3^{\text {rd }}$ mid tarsomere c. $1.4 \mathrm{x}$ longer than wide.

Areolet pentagonal, frontal distance of intercubiti $3 x$ their diameter; $2^{\text {nd }}$ recurrent vein distinctly distal to its middle. Nervulus postfurcal by $2 \mathrm{x}$ its width.

Metasoma semi-amblypygous, hypopygium moderately elongate (about as long as the distance of its hind margin to metasomal apex). Postpetiolus strongly widened and completely aciculate, lateral field $0.6 \mathrm{x}$ as wide as the median one. $2^{\text {nd }}$ tergite $1.3 \mathrm{x}$ wider than long. Gastrocoelus impressed, with strong ridges; thyridium slightly oblique, $0.8 \mathrm{x}$ as wide as the interval. $2^{\text {nd }}$ and $3^{\text {rd }}$ tergites granulate and with dense superficial punctures, \pm matte; $2^{\text {nd }}$ tergite finely aciculate medially. Following tergites finely granulate, shining. $2^{\text {nd }}$ to $4^{\text {th }}$ sternites with median folds. Ovipositor sheath not extending behind metasomal apex.

Color: Black. Frontal orbit with narrow yellowish stripe. Stripes on flagellomeres 6-14, collare and upper margin of pronotum, subtegular ridge, scutellum, narrow apical band on $2^{\text {nd }}$ tergite (slightly widened medially), small median spot on $4^{\text {th }}$ tergite, and larger roundish spots on $5^{\text {th }}$ to $7^{\text {th }}$ tergites ivory. Legs black; fore tibia and tarsus reddish frontally; mid and hind tibiae with subbasal reddish-brown stripes. Wings with slight yellowish tint; pterostigma ochreous.

$\mathrm{o}^{\Uparrow}$ : unknown.

Remark: This new species belongs to group G2 sensu Hilpert (1992), but differs from all known species of 

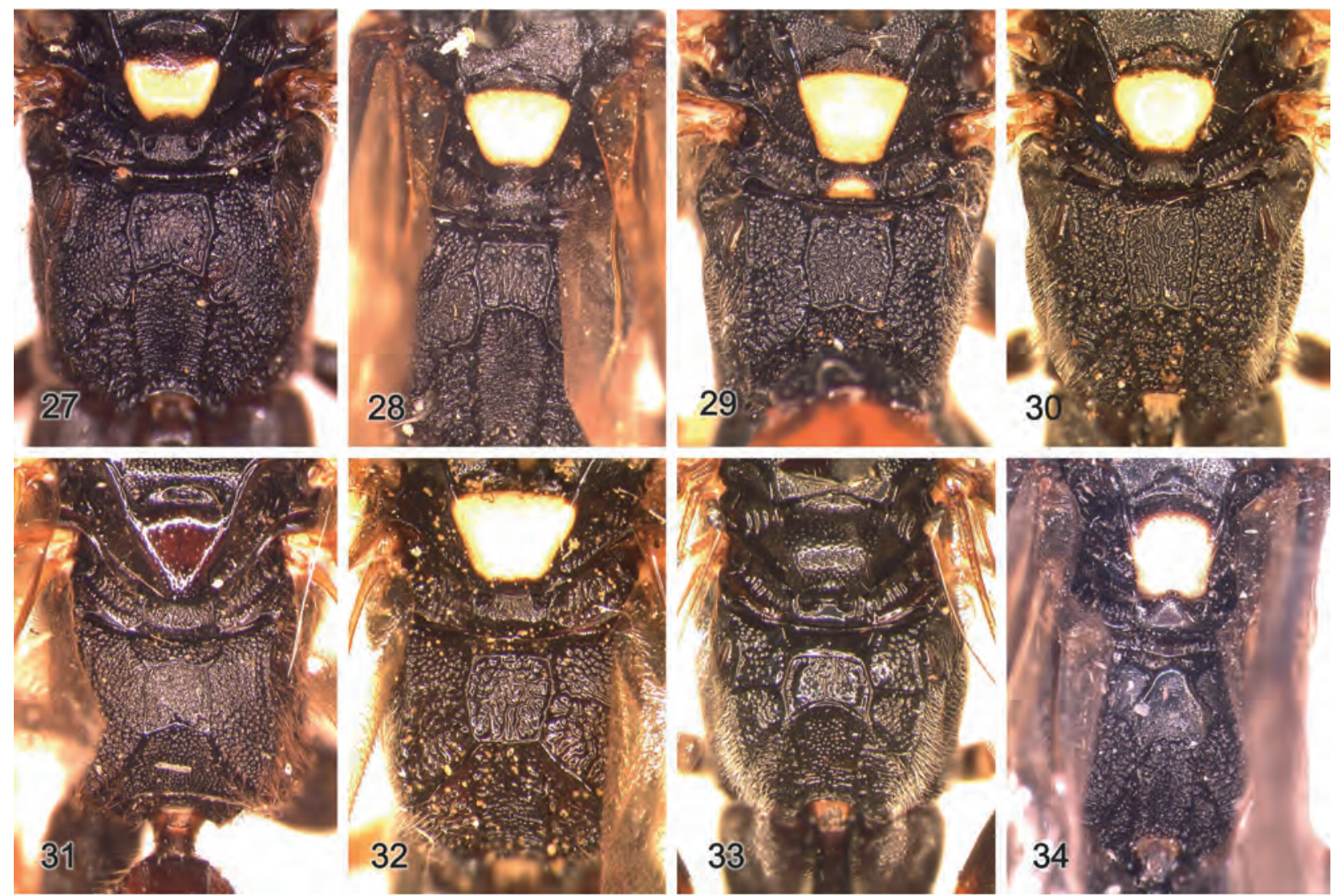

Figs 27-34: Scutellum and propodeum: 27. Ichneumon brevimaculatus spec. nov. $+: 28$. Ichneumon flavocoxalis spec. nov. 29. Ichneumon polyxanthus (Kriechbaumer) + , 30. Ichneumon megextentensorius spec. nov. + , 31. Ichneumon pilocephalus spec. nov. + , 32. Ichneumon flavotibialis spec. nov. + , 33. Monontos baikalensis spec. nov. + : 34. Protichneumon nigriventris spec. nov.

this group by the typical coloration of metasoma with an apical ivory band on $2^{\text {nd }}$ tergite. This color pattern resembles Ichneumon rudolphi Holmgren which has been reported from Kamtschatka (Roman, 1927: 11), but the new taxon differs from I. rudolphi by its larger size, smaller thyridium, extended ivory coloration of mesosoma, ivory spots on $4^{\text {th }}$ and $5^{\text {th }}$ tergites, and dark tibiae without yellow rings.

\section{Monontos baikalensis spec. nov.}

urn:Isid:zoobank.org:act:FD6CE0F9-611F-4590-9511-6DBABF35EE9A (Figs 33, 40-42)

Holotype: + "Russia E Siberia, lake Baikal near Anghasolka, 6.IV.2007, leg. Berlov" "under bark of Pinus silvestris" (coll. Riedel).

Description: + . Body length $12.3 \mathrm{~mm}$. Flagellum almost filiform, with 37 flagellomeres; $1^{\text {st }}$ flagellomere $3.3 \mathrm{x}$ longer than wide, c. $12^{\text {th }}$ flagellomere square, widest flagellomeres c. $1.25 \mathrm{x}$ wider than long; preapical flagellomere square. Temple shortly parallel behind eye, moderately and roundly narrowed apcially, dorsally $0.4 \mathrm{x}$ as long as eye. Distance of lateral ocellus to eye $1.3 \mathrm{x}$ ocellar diameter. Frons rugose-punctate and granulate dorsally, smooth above antennal sockets. Face densely punctate, granulate, matte. Clypeus large, $2.1 \mathrm{x}$ wider than long, flat and slightly impressed centrally, with scattered punctures, shining; apical margin sharp, slightly concave medially. Gena swollen, with dense punctures ventrally. Malar space $0.9 \mathrm{x}$ as long as width of mandibular base. Mandible sickle-shaped, with long upper tooth, lower tooth not indicated. Genal carina complete, slightly curved inwards ventrally and reaching mandibular base, low. Hypostomal carina moderately lamelliform widened (about as wide as distal maxillary palp).

Mesosoma covered with whitish hairs. Notaulus impressed at frontal margin of mesoscutum. Mesoscutum densely punctate and granulate, matte. Mesopleuron and metapleuron densely rugose-punctate; speculum partly smooth; juxtacoxal carina present. Scutellum distinctly elevated, $1.3 \mathrm{x}$ wider than long, densely punctate, with lateral carina in basal half. Propodeum completely carinate, moderately sloping apically, but area superomedia on an almost horizontal plane. Area basalis with median tubercle. Area superomedia hexagonal, but concave apically, $1.2 \mathrm{x}$ wider than long, rugose; costula reaching its middle. Area petiolaris transversely rugose, with lateral carina. Hind coxa densely punctate, without scopa. Hind femur with dense punctures, 4.0x longer than wide. Claws not pectinate. 

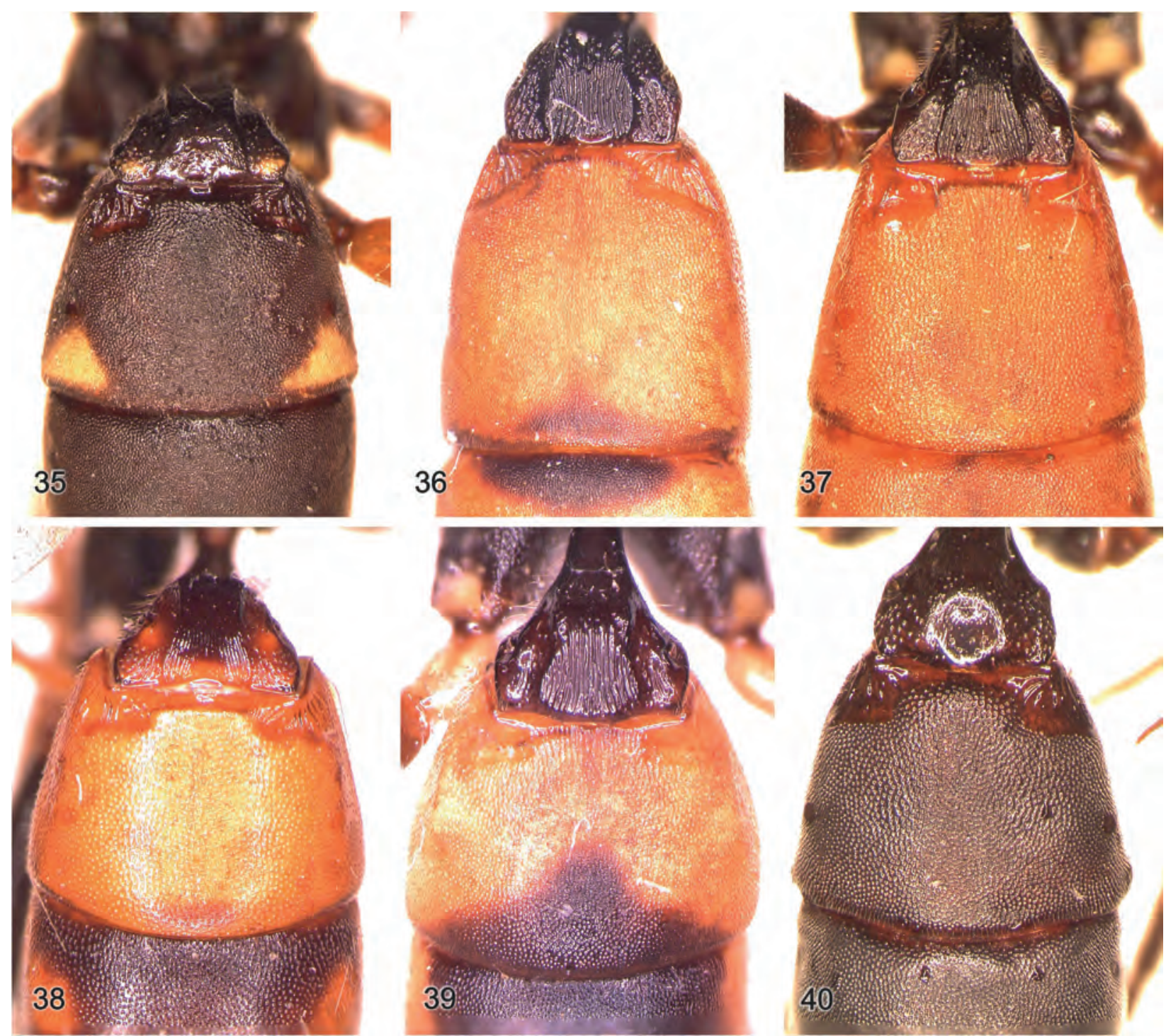

Figs 35-40: Basal tergites of: 35. Ichneumon brevimaculatus spec. nov. + , 36. Ichneumon polyxanthus (KRIECHBAUMER) + , 37. Ichneumon megextensorius spec. nov. ㅇ, 38. Ichneumon pilocephalus spec. nov. ㅇ, 39. Ichneumon flavotibialis spec. nov., 40. Monontos baikalensis spec. nov.

Areolet pentagonal, frontal distance of intercubiti $4 \mathrm{x}$ their width; $2^{\text {nd }}$ recurrent vein slightly distal to its middle. Nervulus interstitial.

Metasoma oxypygous. Postpetiolus strongly widened, without dorsal carina; field not clearly separated, lateral field with dense punctures, wide median field with very fine partly longitudinal aciculation and small punctures. $2^{\text {nd }}$ tergite $1.25 \mathrm{x}$ wider than long. Gastrocoelus distinctly impressed, with strong ridges; thyridium slightly oblique, c. $0.7 \mathrm{x}$ as wide as the interval. $2^{\text {nd }}$ and $3^{\text {rd }}$ tergites densely rugose-punctate, \pm matte; $4^{\text {th }}$ tergite with superficial punctures, \pm shining. Ovipositor sheath distinctly extending behind metasomal apex (by length of $3^{\text {rd }}$ hind tarsomere).

Color: Black. Flagellomeres 9-13, narrow frontal orbit and narrow stripes on vertex ivory. Legs black; bases of femora narrowly reddish; fore femur apically and fore tibia frontally \pm yellowish. Wings hyaline; pterostigma brown. $o^{*}$ : unknown.
Remark: The new taxon is related to Monontos niphonicus UCHIDA, 1926 and can be distinguished by its wider temple and gena, ventrally curved genal carina and widened hypostomal carina, entirely black mesosoma, and matte basal tergites.

The genus Monontos UCHIDA is actually placed in the tribe Heresiarchini (Yu et al., 2016) due to the sloping form of propodeum. As it is found in related genera such as Syspasis Townes or Orgichneumon HeInRICH, the large and usually halfmoon-shaped area superomedia is almost plane and horizontal and not rounded in profile as in Coelichneumon and several other typical genera of the tribe Heresiarchini. In addition, the collection date and location of this new species indicates an overwintering. This biological behaviour has also found in Orgichneumon (see below) and other genera of Ichneumonini but not in typical Heresiarchini. I therefore transfer the genus Monontos Uchida to the tribe Ichneumonini. 


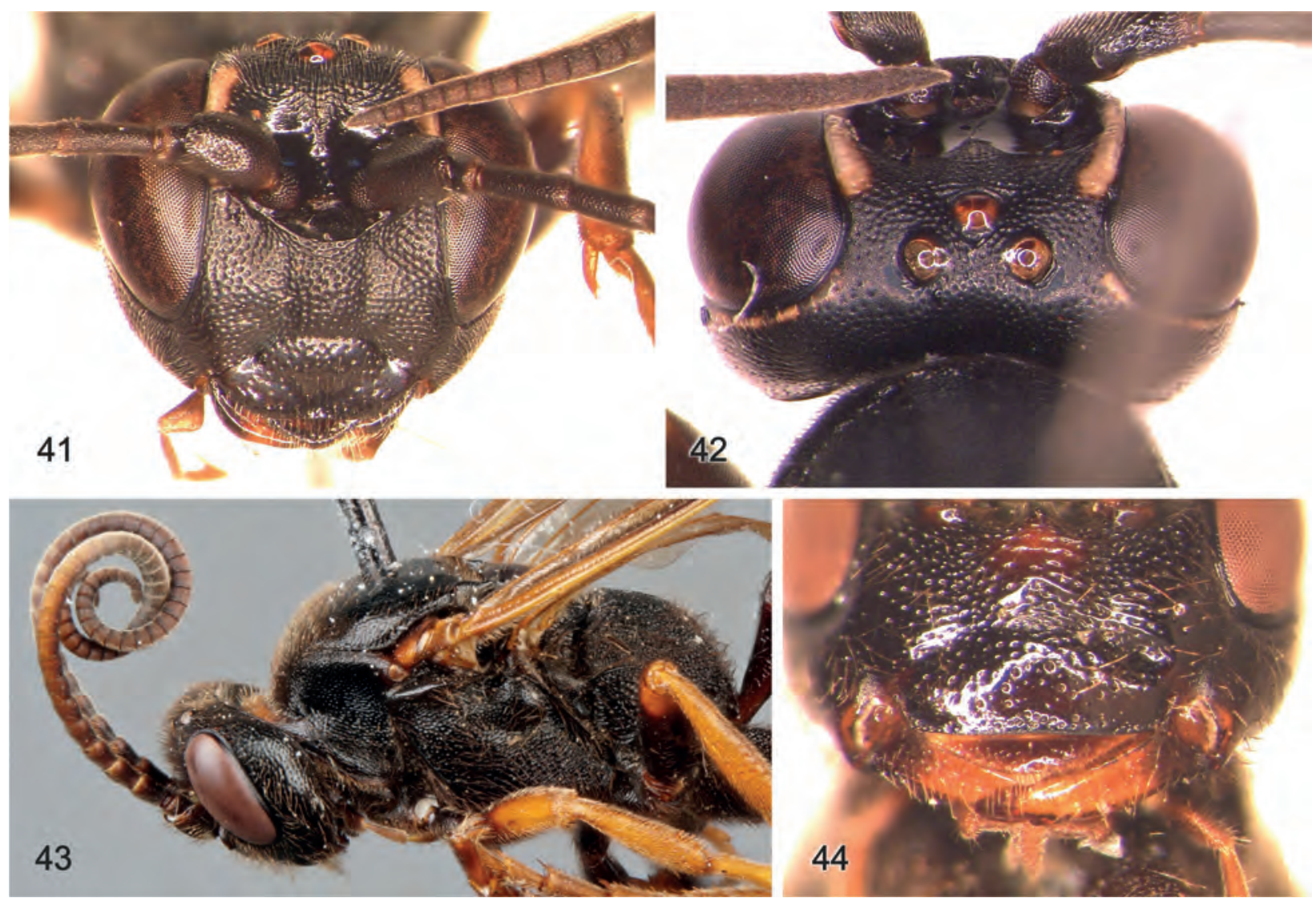

Figs 41-42: Monontos baikalensis spec. nov. 우 : 41. face, 42. Head from dorsal.- Figs 43-44: Ichneumon pilocephalus spec. nov. 오 43. Habitus from lateral, 44. mandible.

\section{Orgichneumon calcatorius (ThUNBERG, 1824)}

Material: East Siberia, Lake Baikal near Anghasolka, under bark, 3 ㅇ ㅇ 2007-04-06, leg. Berlov (coll. Riedel).

Distribution: Holarctic region, new record for Siberia.

\section{Patrocloides dubitatorius (SULZER, 1776)}

Material: Russia Far East, Primorskiy kray, Anisimovka, Litovka Mountains, $70 \mathrm{~km}$ East of Vladivostok, $43^{\circ} 07^{\prime} \mathrm{N}$ $132^{\circ} 41^{\prime}$ E, 900 m, 1 ㅇ 1993-06-08, leg. A. Taeger (SDEI); Russia Far East, Primorskij Kray, Ryazanovska, 14 km Southwest of Slavyanka, $42^{\circ} 48^{\prime} \mathrm{N} 131^{\circ} 12^{\prime} \mathrm{E}, 50 \mathrm{~m}, 1$ 우, 1 o 1993-06-16, leg. Taeger (SDEI).

Distribution: Palaearctic region, known from Siberia (Roman, 1904).

\section{Patrocloides dubitatorius forma rufator}

Material: Russia Far East, Primorskiy kray, Przhevalski Mountains, $53 \mathrm{~km}$ Southeast of Ussuriysk, $43^{\circ} 37^{\prime} \mathrm{N}$ $132^{\circ} 35^{\prime}, 250 \mathrm{~m}, 1$ 우 1993-06-13, leg. A. Taeger (SDEI).
Remark: This color form is characterized by its mainly rufous mesosoma and metasoma with some blackish suffusion. Yellow are flagellomeres 8-12, facial orbit, collare and upper margin of pronotum, subtegular ridge, scutellum posterolateral spots on $1^{\text {st }}$ and $2^{\text {nd }}$ tergites, frontal stripe on fore femur, subbasal rings of all tibiae and of $1^{\text {st }}$ and $2^{\text {nd }}$ tarsomeres.

\section{Patrocloides regius (HELLÉN, 1951)}

Material: East Siberia, Irkutsk, $1 \sigma^{\pi}$ 2000-07-16, leg. Berlov (coll. Riedel).

Distribution: Known from Finland and Russia including Siberia (Vikberg \& Perkiomaki, 2006).

\section{Probolus crassulus HoRSTMANN, 2000}

Material: Southwest Siberia, Orenburg region, Ilek surrounds, 1 o $2005-08-01$ to 2005-08-05, leg. Osipov (coll. Riedel).

Distribution: West Palaearctic region including Iran, new record for Siberia. 


\section{Spilichneumon occisorius (FABRICIUS, 1793)}

Material: South Siberia, Southwest Tannu-Ola mountain range, Southwest Tuwa region, Sogly surrounds, $2000 \mathrm{~m}$, 26 우 2003-07-01 to 2003-07-05, leg. Vastchenko (coll. Riedel).

Distribution: West Palaearctic region including Iran, new record for Siberia.

\section{Stenichneumon militarius (THUNBERG, 1824)}

Material: Russia, East Siberia, 10 km East of Irkutsk, 1 ㅇ 2003-09-28, leg. Berlov (coll. Riedel).

Distribution: Holarctic region, known from Siberia (HEINRICH, 1978).

\section{Stenichneumon ussuriensis HeINRICH, 1980}

Material: Russia Far East, Primorskiy kray, Banevurovo, South of Ussuriysk, $43^{\circ} 42^{\prime} \mathrm{N} 132^{\circ} 01^{\prime} \mathrm{E}, 150 \mathrm{~m}$, 1 i 1993-06-10, leg. A. Taeger (SDEI).

Remark: The available specimen differs from the holotype by: Body length $14 \mathrm{~mm}$; flagellum with $38 \mathrm{flag}$ ellomeres; $1^{\text {st }}$ flagellomere $4.0 \mathrm{x}$ longer than wide; hind femur $4.3 \mathrm{x}$ longer than wide. The specimen is otherwise typical.

Distribution: Only known from Russia Far East (HeINRICH, 1980).

\section{Triptognathus atripes (GRAVENHORST, 1820)}

Material: Russia Far East, Primorskiy kray, SikhoteAlin, Oblachnaya $56 \mathrm{~km}$ Southeast of Chuguyevka, $43^{\circ} 45^{\prime} \mathrm{N} 134^{\circ} 15^{\prime} \mathrm{E}, 850 \mathrm{~m}, 2$ 우 우 1992-06-02, leg. A. Taeger (SDEI).

Distribution: Western Palaearctic region and Japan; new record for Far East Russia.

\section{Virgichneumon albilineatus (GravenHORST, 1820)}

Material: Russia Far East, Primorskiy Kray, Lazovski Zapovednik, c. $170 \mathrm{~km}$ East of Vladivostok, Ta-Chingousa, $43^{\circ} 01^{\prime} 07^{\prime \prime} \mathrm{N} 134^{\circ} 07^{\prime} 46^{\prime \prime} \mathrm{E}, 0 \mathrm{~m}, 2$ o $^{\star}$ o $^{\star}$ 2001-08-31 to 2001-09-16, sandy coast, Malaise trap 495, leg. M. Quest (NHM).

Distribution: Palaearctic and Oriental regions; known from Russia Far East (Meyer, 1933).

\section{Vulgichneumon leucaniae (UCHIDA, 1924)}

Material: Russia Far East, Primorskiy Kray, Lazovski Zapovednik, c. $170 \mathrm{~km}$ East of Vladivostok, Ta-Chingousa, $43^{\circ} 01^{\prime} 07^{\prime \prime} \mathrm{N} 134^{\circ} 07^{\prime} 46^{\prime \prime} \mathrm{E}, 0 \mathrm{~m}, 10^{\star}$ 2001-08-31 to 2001-09-16, sandy coast, Malaise trap 495, leg. M. Quest (NHM).

Distribution: East Palaearctic and Oriental regions, known from Russia Far East (UCHIDA, 1926).

\section{Vulgichneumon saturatorius (LINNAEUS, 1758)}

Material: Russia Far East, Primorskiy Kray, Lazovski Zapovednik, c. $170 \mathrm{~km}$ East of Vladivostok, Korpad, $43^{\circ} 15^{\prime} 52^{\prime \prime} \mathrm{N} 134^{\circ} 07^{\prime} 45^{\prime \prime} \mathrm{E}, 174 \mathrm{~m}, 1$ o $^{\top} 2001-05-28$ to 2001-06-06, flood plain, Malaise trap 453, leg. M. Quest (NHM)

Distribution: Palaearctic region, known from Japan and Siberia (Meyer, 1933).

\section{Zanthojoppa lutea (GRAVENHORST, 1829)}

Material: Far East Russia, Primorskiy kray, Anisimovka,

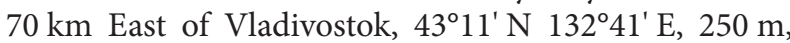
1 ㅇ 1993-06-07, leg. A. Taeger (SDEI).

Distribution: Palaearctic region, known from Siberia (HEINRICH, 1978).

\section{Acknowledgements}

I thank A. TAeger (SDEI), St. SChmidt (ZSM), G. BroAd (NHM), M. SHAw (EDI) and M. HARTMANN (Erfurt) for their kind and generous loan of East Palaearctic Ichneumoninae from their institutions. I also thank E. DiLLER (ZSM) for his kind and helpful determination of the Phaeogeninae. Additionally, I'd like to thank all collectors who donated their interesting material of Ichneumoninae to my personal collection, especially O. BERLOV (Irkutsk/ Siberia).

\section{References}

Broad, G. R.; Shaw, M. R. \& Fitton, M. G. 2018: Ichneumonid wasps (Hymenoptera: Ichneumonidae): their Classification and Biology. Handbooks for the Identification of British Insects Vol. 7, Part 12. Royal Entomological Society, London: 1-417.

Diller, E. \& Schönitzer, K. 2003: Revision einiger westpaläarktischer und nearktischer Arten der Gattung Colpognathus Wesmael (1845) (Hymenoptera, Ichneumonidae, Ichneumoninae, Alomyini). - Entomofauna 24: 333-342. 
Heinrich, G. H. 1978: Eastern Palearctic Hymenopterous insects of the subfamily Ichneumoninae (in Russian). - Leningrad: 1-81.

HeinRich, G. H. 1980: Neue Ichneumoninae Stenopneusticae aus der paläarktischen Region. - Mitteilungen der Münchner Entomologischen Gesellschaft 69: 9-27.

Hilpert, H. 1992: Zur Systematik der Gattung Ichneumon LinNAEUs, 1758 in der Westpalaearktis. Entomofauna Supplement 6: 1-389.

Lelej, A. S. (Ed.) 2012: Annotated catalogue of the insects of Russian Far East. Volume I. Hymenoptera (In Russian). Vladivostok, Dalauka: 1-635.

Riedel, M. 2008a: Revision der westpaläarktischen Platylabini (Hymenoptera, Ichneumonidae, Ichneumoninae): I. Die Gattung Platylabus Wesmael, 1845. - Spixiana 31: 105-172.

Riedel, M. 2008b: Revision einiger von schwedischen Autoren beschriebenen Arten der Gattung Coelichneumon Thomson (Hymenoptera: Ichneumonidae, Ichneumoninae). - Entomofauna 29: 185-196.

Riedel, M. 2012: Revision der westpaläarktischen Arten der Gattung Coelichneumon Thomson (Hymenoptera, Ichneumonidae). - Linzer Biologische Beiträge 44: 1477-1611.

Riedel, M. 2018: Contribution to the Siberian species of Ichneumon LinnaEus (Hymenoptera, Ichneumonidae, Ichneumoninae). - Linzer Biologische Beiträge 50: 1509-1562.

Roman, A. 1904: Sibirische Ichneumonen im Schwedischen Reichsmuseum. - Entomologisk Tidskrift 25: 138-150.

Roman, A. 1927: Entomologische Ergebnisse der schwedischen Kamtschatka-Expedition 1920-1922. 10. Ichneumonidae, Subfam. Ichneumoninae. - Arkiv för Zoologi 19A (7): 1-19.

SiYtan, U. V. 1977: A review of the tribe Phaeogenini (Hymenoptera, Ichneumonidae) in the European regions of the USSR. - Entomological Review 56: 93-100.
Tereshrin, A. 2003: Ichneumon flies of the genus Cratichneumon (Hymenoptera, Ichneumonidae) in the forest zone. 1 Taxonomic notes. Description of Cratichneumon unificatus sp. n. (in Russian). Zoological Zhurnal 82: 594-602.

Tosquinet, J. 1889: Documents pour servir à la monographie des Ichneumonides de la Russie d’Asie. - Annales de la Société Entomologique de Belgique 33: $125-148$.

Townes, H.; MomoI, S. \& Townes, M. 1965: A catalogue and reclassification of the Eastern Palearctic Ichneumonidae. - Memoirs of the American Entomological Institute 5: 1-661.

UchidA, T. 1926: Erster Beitrag zur IchneumonidenFauna Japans. - Journal of the Faculty of Agriculture, Hokkaido Imperial University 18: 43-173.

UCHIDA, T. 1935: Zur Ichneumonidenfauna von Tosa (I.) Subfam. Ichneumoninae. - Insecta Matsumurana 10: 6-33.

UchidA, T. 1936: Drei neue Gattungen sowie acht neue und fünf unbeschriebene Arten der Ichneumoniden aus Japan. - Insecta Matsumurana 10: 111-122.

UchidA, T. 1941: Die Kriechbaumer Typen der japanischen Ichneumoniden. - Transactions of the Sapporo Natural History Society 16: 227-230.

Vikberg, V. \& Perkiomaki, J. 2006: Patrocloides regius (Hellén, 1951), an extinct species in Finnish fauna? (Hymenoptera: Ichneumonidae: Ichneumoninae). Sahlbergia 11: 14-20.

WoldstedT, F. W. 1881: Fundorte russischer Ichneumoniden. - Horae Societatis Entomologicae Rossicae 16: 58-64.

Yu, D.; van Achterberg, C. \& Horstmann, K. 2016: Taxapad 2016 - World Ichneumonoidea 2015. Taxonomy, Biology, Morphology and Distribution. Nepean, Ontario, Canada. 